\title{
Conditioned Brownian trees
}

\author{
Jean-François Le Gall *, Mathilde Weill \\ DMA - Ecole normale supérieure de Paris, 45, rue d'Ulm, 75005 Paris, France
}

Received 30 November 2004; accepted 30 August 2005

Available online 19 January 2006

\begin{abstract}
We consider a Brownian tree consisting of a collection of one-dimensional Brownian paths started from the origin, whose genealogical structure is given by the Continuum Random Tree (CRT). This Brownian tree may be generated from the Brownian snake driven by a normalized Brownian excursion, and thus yields a convenient representation of the so-called Integrated SuperBrownian Excursion (ISE), which can be viewed as the uniform probability measure on the tree of paths. We discuss different approaches that lead to the definition of the Brownian tree conditioned to stay on the positive half-line. We also establish a Vervaatlike theorem showing that this conditioned Brownian tree can be obtained by re-rooting the unconditioned one at the vertex corresponding to the minimal spatial position. In terms of ISE, this theorem yields the following fact: Conditioning ISE to put no mass on ] $-\infty,-\varepsilon$ [ and letting $\varepsilon$ go to 0 is equivalent to shifting the unconditioned ISE to the right so that the left-most point of its support becomes the origin. We derive a number of explicit estimates and formulas for our conditioned Brownian trees. In particular, the probability that ISE puts no mass on $]-\infty,-\varepsilon$ [ is shown to behave like $2 \varepsilon^{4} / 21$ when $\varepsilon$ goes to 0 . Finally, for the conditioned Brownian tree with a fixed height $h$, we obtain a decomposition involving a spine whose distribution is absolutely continuous with respect to that of a nine-dimensional Bessel process on the time interval $[0, h]$, and Poisson processes of subtrees originating from this spine.
\end{abstract}

(c) 2005 Elsevier SAS. All rights reserved.

\section{Résumé}

Nous considérons un arbre brownien formé par une famille de trajectoires browniennes issues de l'origine sur la droite, dont la structure généalogique est le Continuum Random Tree (CRT). Cet arbre brownien peut être construit à partir du serpent brownien dirigé par une excursion brownienne normalisée, et fournit une représentation simple de la mesure aléatoire appelée ISE, qui peut être vue comme la probabilité uniforme sur l'arbre des trajectoires. Nous discutons différentes approches qui conduisent à la définition de l'arbre brownien conditionné à rester du coté positif. Nous établissons aussi un théorème à la Vervaat qui montre que cet arbre brownien conditionné peut être obtenu en réenracinant l'arbre non conditionné au sommet donnant la position spatiale la plus à gauche. Pour la mesure ISE, cela donne le résultat suivant : conditionner ISE à ne pas charger l'intervalle ] $-\infty,-\varepsilon$ [ et faire tendre $\varepsilon$ vers 0 revient à translater la mesure non conditionnée vers la droite de façon que l'origine devienne le point le plus à gauche de son support. Nous donnons des estimations et des formules explicites pour l'arbre brownien conditionné. En particulier, la probabilité que ISE ne charge pas l'intervalle $]-\infty,-\varepsilon$ [ se comporte comme $2 \varepsilon^{4} / 21$ quand $\varepsilon$ tend vers 0 . Finalement, pour l'arbre brownien conditionné avec une hauteur $h$, nous obtenons une décomposition combinant une arête principale, de loi absolument continue par rapport à celle d'une trajectoire de processus de Bessel de dimension 9 sur l'intervalle $[0, h]$, avec un processus de Poisson de sous-arbres issus de cette arête principale.

(c) 2005 Elsevier SAS. All rights reserved.

\footnotetext{
* Corresponding author. Tel.: +(33) 1443231 68; fax: +(33) 144322080 .

E-mail addresses: legall@dma.ens.fr (J.-F. Le Gall), weill@dma.ens.fr (M. Weill).
} 
MSC: 60J80; 60J65; 60G57

Keywords: Random tree; CRT; ISE; Conditioned tree; Brownian snake; Re-rooting

\section{Introduction}

In this work, we define and study a continuous tree of one-dimensional Brownian paths started from the origin, which is conditioned to remain in the positive half-line. An important motivation for introducing this object comes from its relation with analogous discrete models which are discussed in several recent papers.

In order to present our main results, let us briefly describe a construction of unconditioned Brownian trees. We start from a positive Brownian excursion conditioned to have duration 1 (a normalized Brownian excursion in short), which is denoted by $(e(s), 0 \leqslant s \leqslant 1)$. This random function can be viewed as coding a continuous tree via the following simple prescriptions. For every $s, s^{\prime} \in[0,1]$, we set

$$
m_{e}\left(s, s^{\prime}\right):=\inf _{s \wedge s^{\prime} \leqslant r \leqslant s \vee s^{\prime}} e(r) .
$$

We then define an equivalence relation on $[0,1]$ by setting $s \sim s^{\prime}$ if and only if $e(s)=e\left(s^{\prime}\right)=m_{e}\left(s, s^{\prime}\right)$. Finally we put

$$
d_{e}\left(s, s^{\prime}\right)=e(s)+e\left(s^{\prime}\right)-2 m_{e}\left(s, s^{\prime}\right)
$$

and note that $d_{e}\left(s, s^{\prime}\right)$ only depends on the equivalence classes of $s$ and $s^{\prime}$. Then the quotient space $\mathbf{T}_{e}:=[0,1] / \sim$ equipped with the metric $d_{e}$ is a compact $\mathbb{R}$-tree (see e.g. Section 2 of [15]). In other words, it is a compact metric space such that for any two points $\sigma$ and $\sigma^{\prime}$ there is a unique arc with endpoints $\sigma$ and $\sigma^{\prime}$ and furthermore this arc is isometric to a compact interval of the real line. We view $\mathbf{T}_{e}$ as a rooted $\mathbb{R}$-tree, whose root $\rho$ is the equivalence class of 0 . For every $\sigma \in \mathbf{T}_{e}$, the ancestral line of $\sigma$ is the line segment joining $\rho$ to $\sigma$. This line segment is denoted by $\llbracket \rho, \sigma \rrbracket$. We write $\dot{s}$ for the equivalence class of $s$, which is a vertex in $\mathbf{T}_{e}$ at generation $e(s)=d_{e}(0, s)$.

Up to unimportant scaling constants, $\mathbf{T}_{e}$ is the Continuum Random Tree (CRT) introduced by Aldous [3]. The preceding presentation is indeed a reformulation of Corollary 22 in [5], which was proved via a discrete approximation (a more direct approach was given in [22]). As Aldous [5] has shown, the CRT is the scaling limit of critical GaltonWatson trees conditioned to have a large fixed progeny (see [14] and [15] for recent generalizations of Aldous' result). The fact that Brownian excursions can be used to model continuous genealogies had been used before, in particular in the Brownian snake approach to superprocesses (see [21]).

We can now combine the branching structure of the CRT with independent spatial motions. We restrict ourselves to spatial displacements given by linear Brownian motions, which is the case of interest in this work. Conditionally given $e$, we introduce a centered Gaussian process $\left(V_{\sigma}, \sigma \in \mathbf{T}_{e}\right)$ with covariance

$$
\operatorname{cov}\left(V_{\dot{s}}, V_{\dot{s}^{\prime}}\right)=m_{e}\left(s, s^{\prime}\right), \quad s, s^{\prime} \in[0,1] .
$$

This definition should become clear if we observe that $m_{e}\left(s, s^{\prime}\right)$ is the generation of the most recent common ancestor to $\dot{s}$ and $\dot{s}^{\prime}$ in the tree $\mathbf{T}_{e}$. It is easy to verify that the process $\left(V_{\sigma}, \sigma \in \mathbf{T}_{e}\right)$ has a continuous modification. The random measure $\mathcal{Z}$ on $\mathbb{R}$ defined by

$$
\langle\mathcal{Z}, \varphi\rangle=\int_{0}^{1} \varphi\left(V_{\dot{s}}\right) \mathrm{d} s
$$

is then the one-dimensional Integrated Super-Brownian Excursion (ISE, see Aldous [6]). Note that ISE in higher dimensions, and related Brownian trees, have appeared recently in various asymptotic results for statistical mechanics models (see e.g. [13,17,29]). The support, or range, of ISE is

$$
\mathcal{R}:=\left\{V_{\sigma}: \sigma \in \mathbf{T}_{e}\right\} .
$$

For our purposes, it is also convenient to reinterpret the preceding notions in terms of the Brownian snake. The Brownian snake $\left(W_{s}, 0 \leqslant s \leqslant 1\right)$ driven by the normalized excursion $e$ is obtained as follows (see Subsection 2.1 for a more detailed presentation). For every $s \in[0,1], W_{s}=\left(W_{s}(t), 0 \leqslant t \leqslant e(s)\right)$ is the finite path which gives the spatial 
positions along the ancestral line of $\dot{s}: W_{s}(t)=V_{\sigma}$ if $\sigma$ is the vertex at distance $t$ from the root on the segment $\llbracket \rho, \dot{s} \rrbracket$. Note that $W_{s}$ only depends on the equivalent class $\dot{s}$. We view $W_{s}$ as a random element of the space $\mathcal{W}$ of finite paths.

Our first goal is to give a precise definition of the Brownian tree $\left(V_{\sigma}, \sigma \in \mathbf{T}_{e}\right)$ conditioned to remain positive. Equivalently this amounts to conditioning ISE to put no mass on the negative half-line. Our first theorem gives a precise meaning to this conditioning in terms of the Brownian snake. We denote by $\mathbb{N}_{0}^{(1)}$ the distribution of $\left(W_{s}, 0 \leqslant\right.$ $s \leqslant 1)$ on the canonical space $C([0,1], \mathcal{W})$ of continuous functions from $[0,1]$ into $\mathcal{W}$, and we abuse notation by still writing $\left(W_{s}, 0 \leqslant s \leqslant 1\right)$ for the canonical process on this space. The range $\mathcal{R}$ is then defined under $\mathbb{N}_{0}^{(1)}$ by

$$
\mathcal{R}=\left\{\widehat{W}_{s}: 0 \leqslant s \leqslant 1\right\}
$$

where $\widehat{W}_{s}$ denotes the endpoint of the path $W_{s}$.

\section{Theorem 1.1. We have}

$$
\lim _{\varepsilon \downarrow 0} \varepsilon^{-4} \mathbb{N}_{0}^{(1)}(\mathcal{R} \subset]-\varepsilon, \infty[)=\frac{2}{21} .
$$

There exists a probability measure on $C([0,1], \mathcal{W})$, which is denoted by $\overline{\mathbb{N}}_{0}^{(1)}$, such that

$$
\lim _{\varepsilon \downarrow 0} \mathbb{N}_{0}^{(1)}(\cdot \mid \mathcal{R} \subset]-\varepsilon, \infty[)=\overline{\mathbb{N}}_{0}^{(1)},
$$

in the sense of weak convergence in the space of probability measures on $C([0,1], \mathcal{W})$.

Our second theorem gives an explicit representation of the conditioned measures $\overline{\mathbb{N}}_{0}^{(1)}$, which is analogous to a famous theorem of Vervaat [30] relating the normalized Brownian excursion to the Brownian bridge. To state this result, we need the notion of re-rooting. For $s \in[0,1]$, we write $\mathbf{T}_{e}^{[s]}$ for the "same" tree $\mathbf{T}_{e}$ but with root $\dot{s}$ instead of $\rho=\dot{0}$. We then shift the spatial positions by setting $V_{\sigma}^{[s]}=V_{\sigma}-V_{\dot{s}}$ for every $\sigma \in \mathbf{T}_{e}$, in such a way that the spatial position of the new root is still the origin. (Notice that both $\mathbf{T}_{e}^{[s]}$ and $V^{[s]}$ only depend on $\dot{s}$, and we could as well define $\mathbf{T}_{e}^{[\sigma]}$ and $V^{[\sigma]}$ for $\sigma \in \mathbf{T}_{e}$.) Finally, the re-rooted snake $W^{[s]}=\left(W_{r}^{[s]}, 0 \leqslant r \leqslant 1\right)$ is defined analogously as before: For every $r \in[0,1], W_{r}^{[s]}$ is the path giving the spatial positions $V_{\sigma}^{[s]}$ along the ancestral line (in the re-rooted tree) of the vertex $s+r \bmod 1$.

Theorem 1.2. Let $s_{*}$ be the unique time of the minimum of $\widehat{W}$ on $[0,1]$. The probability measure $\overline{\mathbb{N}}_{0}^{(1)}$ is the law under $\mathbb{N}_{0}^{(1)}$ of the re-rooted snake $W^{\left[s_{*}\right]}$.

If we want to define one-dimensional ISE conditioned to put no mass on the negative half-line, the most natural way is to condition to put no mass on $]-\infty,-\varepsilon$ [ and then to let $\varepsilon$ go to 0 . As a consequence of the previous two theorems, this is equivalent to shifting the unconditioned ISE to the right, so that the left-most point of its support

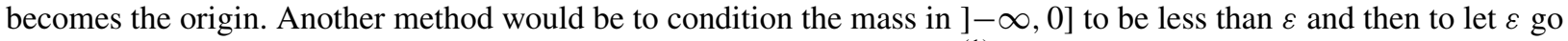
to 0 . Proposition 3.7 below shows that this leads to the same measure $\overline{\mathbb{N}}_{0}^{(1)}$.

Both Theorems 1.1 and 1.2 could be presented in a different and perhaps more elegant manner by using the formalism of spatial trees as in Section 5 of [15]. In this formalism, a spatial tree is a pair $(\mathbf{T}, U)$ where $\mathbf{T}$ is a compact rooted $\mathbb{R}$-tree (in fact an equivalent class of such objects modulo root-preserving isometries) and $U$ is a continuous mapping from $\mathbf{T}$ into $\mathbb{R}^{d}$. Then the second assertion of Theorem $1.1 \mathrm{can}$ be rephrased by saying that the conditional distribution of the spatial tree $\left(\mathbf{T}_{e}, V\right)$ knowing that $\left.\mathcal{R} \subset\right]-\varepsilon, \infty$ [ has a limit when $\varepsilon$ goes to 0 , and Theorem 1.2 says that this limit is the distribution of $\left(\mathbf{T}_{e}^{\left[\sigma_{*}\right]}, V^{\left[\sigma_{*}\right]}\right)$ where $\sigma_{*}$ is the unique vertex minimizing $V$. We have chosen the above presentation because the Brownian snake plays a fundamental role in our proofs and also because the resulting statements are stronger than the ones in terms of spatial trees.

Let us discuss the relationship of the above theorems with previous results. The first assertion of Theorem 1.1 is closely related to some estimates of Abraham and Werner [1]. In particular, Abraham and Werner proved that the probability for a Brownian snake driven by a Brownian excursion of height 1 not to hit the set ] $-\infty,-\varepsilon$ [ behaves like a constant times $\varepsilon^{4}$ (see Section 4 below). The $d$-dimensional Brownian snake conditioned not to exit a domain $D$ was studied by Abraham and Serlet [2], who observed that this conditioning gives rise to a particular instance of the 
Brownian snake with drift. The setting in [2] is different from the present work, in that the initial point of the snake lies inside the domain, and not at its boundary as here. We also mention the paper [19] by Jansons and Rogers, who establish a decomposition at the minimum for a Brownian tree where branchings occur only at discrete times.

An important motivation for the present work came from several recent papers that discuss asymptotics for planar maps. Cori and Vauquelin [11] proved that there exists a bijection between rooted planar quadrangulations and certain discrete trees called well-labeled trees (see also Chassaing and Schaeffer [10] for a more tractable description of this bijection). Roughly, a well-labeled tree consists of a (discrete) plane tree whose vertices are given labels which are positive integers, with the constraints that the label of the root is 1 and the labels of two neighboring vertices can differ by at most 1 . Our conditioned Brownian snake should then be viewed as a continuous model for well-labeled trees. This idea was exploited in [10] and especially in Marckert and Mokkadem [27], where the re-rooted snake $W^{\left[s_{*}\right]}$ appears in the description of the Brownian map, which is the continuous object describing scaling limits of planar quadrangulations. In contrast with the present work, the re-rooted snake $W^{\left[s_{*}\right]}$ is not interpreted in [27] as a conditioned object, but rather as a scaling limit of re-rooted discrete snakes. Closely related models of discrete labeled trees are also of interest in theoretical physics: See in particular [7] and [8]. The article [25], which was motivated by [10] and [27], proves that our conditioned Brownian tree is the scaling limit of discrete spatial trees conditioned to remain positive. To be specific, consider a Galton-Watson tree whose offspring distribution is critical and has (small) exponential moments, and condition this tree to have exactly $n$ vertices (in the special case of the geometric distribution, this gives rise to a tree that is uniformly distributed over the set of plane trees with $n$ vertices). This branching structure is combined with a spatial displacement which is a symmetric random walk with bounded jump size on $\mathbb{Z}$. Assuming that the root is at the origin of $\mathbb{Z}$, the spatial tree is then conditioned to remain on the positive side. According to the main theorem of [25], the scaling limit of this conditioned discrete tree when $n \rightarrow \infty$ leads to the measure $\overline{\mathbb{N}}_{0}^{(1)}$ discussed above. The convergence here, and the precise form of the scaling transformation, are as in Theorem 2 of [18], which discusses scaling limits for unconditioned discrete snakes.

Let us now describe the other contributions of this paper. Although the preceding theorems have been stated for the measure $\mathbb{N}_{0}^{(1)}$, a more fundamental object is the excursion measure $\mathbb{N}_{0}$ of the Brownian snake (see e.g. [24]). Roughly speaking, $\mathbb{N}_{0}$ is obtained by the same construction as above, but instead of considering a normalized Brownian excursion, we now let $e$ be distributed according to the (infinite) Itô measure of Brownian excursions. If $\sigma(e)$ denotes the duration of excursion $e$, we have $\mathbb{N}_{0}^{(1)}=\mathbb{N}_{0}(\cdot \mid \sigma=1)$. It turns out that many calculations are more tractable under the infinite measure $\mathbb{N}_{0}$ than under $\mathbb{N}_{0}^{(1)}$. For this reason, both Theorems 1.1 and 1.2 are proved in Section 3 as consequences of Theorem 3.1, which deals with $\mathbb{N}_{0}$. Motivated by Theorem 3.1 we introduce another infinite measure denoted by $\overline{\mathbb{N}}_{0}$, which should be interpreted as $\mathbb{N}_{0}$ conditioned on the event $\{\mathcal{R} \subset[0, \infty[\}$, even though the conditioning requires some care as we are dealing with infinite measures. In the same way as for unconditioned measures, we have $\overline{\mathbb{N}}_{0}^{(1)}=\overline{\mathbb{N}}_{0}(\cdot \mid \sigma=1)$. Another motivation for considering the measure $\overline{\mathbb{N}}_{0}$ comes from connections with superprocesses: Analogously to Chapter IV of [24] in the unconditioned case, $\overline{\mathbb{N}}_{0}$ could be used to define and to analyze a one-dimensional super-Brownian motion started from the Dirac measure $\delta_{0}$ and conditioned never to charge the negative half-line.

In Section 4, we present a different approach that leads to the same limiting measures. If $H(e)$ stands for the height of excursion $e$, we consider for every $h>0$ the measure $\mathbb{N}_{0}^{h}:=\mathbb{N}_{0}(\cdot \mid H=h)$. In the above construction this amounts to replacing the normalized excursion $e$ by a Brownian excursion with height $h$. By using a famous decomposition theorem of Williams, we can then analyze the behavior of the measure $\mathbb{N}_{0}^{h}$ conditioned on the event that the range does not intersect ] $-\infty,-\varepsilon$ [ and show that it has a limit denoted by $\overline{\mathbb{N}}_{0}^{h}$ when $\varepsilon \rightarrow 0$. The method also provides information about the Brownian tree under $\overline{\mathbb{N}}_{0}^{h}$ : This Brownian tree consists of a spine whose distribution is absolutely continuous with respect to that of the nine-dimensional Bessel process, and as usual a Poisson collection of subtrees originating from the spine, which are Brownian snake excursions conditioned not to hit the negative half-line. The connection with the measures $\overline{\mathbb{N}}_{0}^{(1)}$ and $\overline{\mathbb{N}}_{0}$ is made by proving that $\overline{\mathbb{N}}_{0}^{h}=\overline{\mathbb{N}}_{0}(\cdot \mid H=h)$. Several arguments in this section have been inspired by Abraham and Werner's paper [1]. It should also be noted that a discrete version of the nine-dimensional Bessel process already appears in the paper [9] by Chassaing and Durhuus.

At the end of Section 4, we also discuss the limiting behavior of the measures $\overline{\mathbb{N}}_{0}^{h}$ as $h \rightarrow \infty$. This leads to a probability measure $\overline{\mathbb{N}}_{0}^{\infty}$ that should be viewed as the law of an infinite Brownian snake excursion conditioned to stay positive. We again get a description of the Brownian tree coded by $\overline{\mathbb{N}}_{0}^{\infty}$ in terms of a spine and conditioned Brownian 
snake excursions originating from this spine. Moreover, the description is simpler in the sense that the spine is exactly distributed as a nine-dimensional Bessel process started at the origin.

Section 5 gives an explicit formula for the finite-dimensional marginal distributions of the Brownian tree under $\overline{\mathbb{N}}_{0}$, that is for

$$
\overline{\mathbb{N}}_{0}\left(\int_{] 0, \sigma\left[^{p}\right.} \mathrm{d} s_{1} \cdots \mathrm{d} s_{p} F\left(W_{s_{1}}, \ldots, W_{s_{p}}\right)\right)
$$

where $p \geqslant 1$ is an integer and $F$ is a symmetric nonnegative measurable function on $\mathcal{W}^{p}$. In a way similar to the corresponding result for the unconditioned Brownian snake (see (1) below), this formula involves combining the branching structure of certain discrete trees with spatial displacements. Here however because of the conditioning, the spatial displacements turn out to be given by nine-dimensional Bessel processes rather than linear Brownian motions. In the same way as the finite-dimensional marginal distributions of the CRT can be derived from the analogous formula under the Itô measure (see Chapter III of [24]), one might hope to derive the expression of the finite-dimensional marginals under $\overline{\mathbb{N}}_{0}^{(1)}$ from the case of $\overline{\mathbb{N}}_{0}$. This idea apparently leads to untractable calculations, but we still expect Theorem 5.1 to have useful applications in future work about conditioned trees.

Basic facts about the Brownian snake are recalled in Section 2, which also establishes a few important preliminary results, some of which are of independent interest. In particular, we state and prove a general version of the invariance property of $\mathbb{N}_{0}$ under re-rooting (Theorem 2.3). This result is clearly related to the invariance of the CRT under uniform re-rooting, which was observed by Aldous [4] (and generalized to Lévy trees in Proposition 4.8 of [15]). An equivalent form of Theorem 2.3 already appears as Proposition 4.9 of [27]: see the discussion after the statement of this theorem in Subsection 2.3.

\section{Preliminaries}

In this section, we recall the basic facts about the Brownian snake that we will use later, and we also establish a few important preliminary results. We refer to [24] for a more detailed presentation of the Brownian snake and its connections with partial differential equations. In the first four subsections below, we deal with the $d$-dimensional Brownian snake since the proofs are not more difficult in that case, and the results may have other applications.

\subsection{The Brownian snake}

The ( $d$-dimensional) Brownian snake is a Markov process taking values in the space $\mathcal{W}$ of finite paths in $\mathbb{R}^{d}$. Here a finite path is simply a continuous mapping $\mathrm{w}:[0, \zeta] \rightarrow \mathbb{R}^{d}$, where $\zeta=\zeta_{(\mathrm{w})}$ is a nonnegative real number called the lifetime of $\mathrm{w}$. The set $\mathcal{W}$ is a Polish space when equipped with the distance

$$
d\left(\mathrm{w}, \mathrm{w}^{\prime}\right)=\left|\zeta_{(\mathrm{w})}-\zeta_{\left(\mathrm{w}^{\prime}\right)}\right|+\sup _{t \geqslant 0}\left|\mathrm{w}\left(t \wedge \zeta_{(\mathrm{w})}\right)-\mathrm{w}^{\prime}\left(t \wedge \zeta_{\left(\mathrm{w}^{\prime}\right)}\right)\right| .
$$

The endpoint (or tip) of the path $w$ is denoted by $\widehat{w}$. The range of $w$ is denoted by $w\left[0, \zeta_{(w)}\right]$.

In this work, it will be convenient to use the canonical space $\Omega:=C\left(\mathbb{R}_{+}, \mathcal{W}\right)$ of continuous functions from $\mathbb{R}_{+}$ into $\mathcal{W}$, which is equipped with the topology of uniform convergence on every compact subset of $\mathbb{R}_{+}$. The canonical process on $\Omega$ is then denoted by

$$
W_{s}(\omega)=\omega(s), \quad \omega \in \Omega,
$$

and we write $\zeta_{s}=\zeta_{\left(W_{s}\right)}$ for the lifetime of $W_{s}$.

Let $\mathrm{w} \in \mathcal{W}$. The law of the Brownian snake started from $\mathrm{w}$ is the probability measure $\mathbb{P}_{\mathrm{w}}$ on $\Omega$ which can be characterized as follows. First, the process $\left(\zeta_{s}\right)_{s} \geqslant 0$ is under $\mathbb{P}_{\mathrm{w}}$ a reflected Brownian motion in $\left[0, \infty\left[\right.\right.$ started from $\zeta_{(\mathrm{w})}$. Secondly, the conditional distribution of $\left(W_{s}\right)_{s} \geqslant 0$ knowing $\left(\zeta_{s}\right)_{s} \geqslant 0$, which is denoted by $\Theta_{\mathrm{W}}^{\zeta}$, is characterized by the following properties:

(i) $W_{0}=\mathrm{w}, \Theta_{\mathrm{w}}^{\zeta}$ a.s.

(ii) The process $\left(W_{s}\right)_{s} \geqslant 0$ is time-inhomogeneous Markov under $\Theta_{\mathrm{W}}^{\zeta}$. Moreover, if $0 \leqslant s \leqslant s^{\prime}$, 
- $W_{s^{\prime}}(t)=W_{s}(t)$ for every $t \leqslant m\left(s, s^{\prime}\right):=\inf _{\left[s, s^{\prime}\right]} \zeta_{r}, \Theta_{\mathrm{W}}^{\zeta}$ a.s.

- $\left(W_{s^{\prime}}\left(m\left(s, s^{\prime}\right)+t\right)-W_{s^{\prime}}\left(m\left(s, s^{\prime}\right)\right)\right)_{0 \leqslant t \leqslant \zeta_{s^{\prime}}-m\left(s, s^{\prime}\right)}$ is independent of $W_{s}$ and distributed as a $d$-dimensional Brownian motion started at 0 under $\Theta_{\mathrm{w}}^{\zeta}$.

Informally, the value $W_{s}$ of the Brownian snake at time $s$ is a random path with a random lifetime $\zeta_{s}$ evolving like reflecting Brownian motion in $\left[0, \infty\left[\right.\right.$. When $\zeta_{s}$ decreases, the path is erased from its tip, and when $\zeta_{s}$ increases, the path is extended by adding "little pieces" of Brownian paths at its tip.

Excursion measures play a fundamental role throughout this work. We denote by $n(\mathrm{~d} e)$ the Itô measure of positive Brownian excursions. This is a $\sigma$-finite measure on the space $C\left(\mathbb{R}_{+}, \mathbb{R}_{+}\right)$of continuous functions from $\mathbb{R}_{+}$into $\mathbb{R}_{+}$. We write

$$
\sigma(e)=\inf \{s>0: e(s)=0\}
$$

for the duration of excursion $e$. For $s>0, n_{(s)}$ will denote the conditioned measure $n(\cdot \mid \sigma=s)$. Our normalization of the excursion measure is fixed by the relation

$$
n=\int_{0}^{\infty} \frac{\mathrm{d} s}{2 \sqrt{2 \pi s^{3}}} n_{(s)} .
$$

If $x \in \mathbb{R}^{d}$, the excursion measure $\mathbb{N}_{x}$ of the Brownian snake from $x$ is then defined by

$$
\mathbb{N}_{x}=\int_{C\left(\mathbb{R}_{+}, \mathbb{R}_{+}\right)} n(\mathrm{~d} e) \Theta_{\bar{x}}^{e}
$$

where $\bar{x}$ denotes the trivial element of $\mathcal{W}$ with lifetime 0 and initial point $x$. Alternatively, we can view $\mathbb{N}_{x}$ as the excursion measure of the Brownian snake from the regular point $\bar{x}$. With a slight abuse of notation we will also write $\sigma(\omega)=\inf \left\{s>0: \zeta_{s}(\omega)=0\right\}$ for $\omega \in \Omega$. We can then consider the conditioned measures

$$
\mathbb{N}_{x}^{(s)}=\mathbb{N}_{x}(\cdot \mid \sigma=s)=\int_{C\left(\mathbb{R}_{+}, \mathbb{R}_{+}\right)} n_{(s)}(\mathrm{d} e) \Theta_{\bar{x}}^{e} .
$$

Note that in contrast to the introduction we now view $\mathbb{N}_{x}^{(s)}$ as a measure on $\Omega$ rather than on $C([0, s], \mathcal{W})$. The range $\mathcal{R}=\mathcal{R}(\omega)$ is defined by $\mathcal{R}=\left\{\widehat{W}_{s}: s \geqslant 0\right\}$.

Lemma 2.1. Suppose that $d=1$ and let $x>0$. (i) We have

$$
\left.\left.\mathbb{N}_{x}(\mathcal{R} \cap]-\infty, 0\right] \neq \varnothing\right)=\frac{3}{2 x^{2}} \text {. }
$$

(ii) For every $\lambda>0$,

$$
\mathbb{N}_{x}\left(1-\mathbf{1}_{\{\mathcal{R} \cap]-\infty, 0]=\varnothing\}} \mathrm{e}^{-\lambda \sigma}\right)=\sqrt{\frac{\lambda}{2}}\left(3\left(\operatorname{coth}\left(2^{1 / 4} x \lambda^{1 / 4}\right)\right)^{2}-2\right)
$$

where $\operatorname{coth}(y)=\cosh (y) / \sinh (y)$.

Proof. (i) According to Section VI.1 of [24], the function $\left.\left.u(x)=\mathbb{N}_{x}(\mathcal{R} \cap]-\infty, 0\right] \neq \varnothing\right)$ solves $u^{\prime \prime}=4 u^{2}$ in ]0, $\infty$, with boundary condition $u(0+)=+\infty$. The desired result follows.

(ii) See Lemma 7 in [12].

\subsection{Finite-dimensional marginal distributions}

In this subsection we state a result giving information about the joint distribution of the values of the Brownian snake at a finite number of times and its range. In order to state this result, we need some formalism for trees. We first introduce the set of labels

$$
\mathcal{U}=\bigcup_{n=0}^{\infty}\{1,2\}^{n}
$$


where by convention $\{1,2\}^{0}=\{\varnothing\}$. An element of $\mathcal{U}$ is thus a sequence $u=u^{1} \ldots u^{n}$ of elements of $\{1,2\}$, and we set $|u|=n$, so that $|u|$ represents the "generation" of $u$. In particular, $|\varnothing|=0$. The mapping $\pi: \mathcal{U} \backslash\{\varnothing\} \rightarrow \mathcal{U}$ is defined by $\pi\left(u^{1} \ldots u^{n}\right)=u^{1} \ldots u^{n-1}(\pi(u)$ is the "father" of $u)$. In particular, if $k=|u|$, we have $\pi^{k}(u)=\varnothing$.

A binary (plane) tree $\mathcal{T}$ is a finite subset of $\mathcal{U}$ such that:

(i) $\varnothing \in \mathcal{T}$.

(ii) $u \in \mathcal{T} \backslash\{\varnothing\} \Rightarrow \pi(u) \in \mathcal{T}$.

(iii) For every $u \in \mathcal{T}$, either $u 1 \in \mathcal{T}$ and $u 2 \in \mathcal{T}$, or $u 1 \notin \mathcal{T}$ and $u 2 \notin \mathcal{T}$ ( $u$ is called a leaf in the second case).

We denote by $\mathbf{A}$ the set of all binary trees. A marked tree is then a pair $\left(\mathcal{T},\left(h_{u}\right)_{u \in \mathcal{T}}\right)$ where $\mathcal{T} \in \mathbf{A}$ and $h_{u} \geqslant 0$ for every $u \in \mathcal{T}$. We denote by $\mathbb{T}$ the space of all marked trees. In this work it will be convenient to view marked trees as $\mathbb{R}$-trees in the sense of [15] or [16] (see also Section 1 above). This can be achieved through the following explicit construction. Let $\theta=\left(\mathcal{T},\left(h_{u}\right)_{u \in \mathcal{T}}\right)$ be a marked tree and let $\mathbb{R}^{\mathcal{T}}$ be the vector space of all mappings from $\mathcal{T}$ into $\mathbb{R}$. Write $\left(\varepsilon_{u}, u \in \mathcal{T}\right)$ for the canonical basis of $\mathbb{R}^{\mathcal{T}}$. Then consider the mapping

$$
p_{\theta}: \bigcup_{u \in \mathcal{T}}\{u\} \times\left[0, h_{u}\right] \longrightarrow \mathbb{R}^{\mathcal{T}}
$$

defined by

$$
p_{\theta}(u, \ell)=\sum_{k=1}^{|u|} h_{\pi^{k}(u)} \varepsilon_{\pi^{k}(u)}+\ell \varepsilon_{u} .
$$

As a set, the $\mathbb{R}$-tree associated with $\theta$ is the range $\tilde{\theta}$ of $p_{\theta}$. Note that this is a connected union of line segments in $\mathbb{R}^{\mathcal{T}}$. It is equipped with the distance $d_{\theta}$ such that $d_{\theta}(a, b)$ is the length of the shortest path in $\tilde{\theta}$ going from $a$ to $b$. By definition, the range of this path is the segment between $a$ and $b$ and is denoted by $\llbracket a, b \rrbracket$. Finally, we will write $\mathcal{L}_{\theta}$ for (one-dimensional) Lebesgue measure on $\tilde{\theta}$.

By definition, leaves of $\tilde{\theta}$ are points of the form $p_{\theta}\left(u, h_{u}\right)$ where $u$ is a leaf of $\theta$. Points of the form $p_{\theta}\left(u, h_{u}\right)$ when $u$ is not a leaf are called nodes of $\tilde{\theta}$. We write $L(\theta)$ for the set of leaves of $\tilde{\theta}$, and $I(\theta)$ for the set of its nodes. The root of $\tilde{\theta}$ is just the point $0=p_{\theta}(\varnothing, 0)$.

We will consider Brownian motion indexed by $\tilde{\theta}$, with initial point $x \in \mathbb{R}^{d}$. Formally, we may consider, under the probability measure $Q_{x}^{\theta}$, a collection $\left(\xi^{u}\right)_{u \in \mathcal{T}}$ of independent $d$-dimensional Brownian motions all started at 0 except $\xi^{\varnothing}$ which starts at $x$, and define a continuous process $\left(V_{a}, a \in \tilde{\theta}\right)$ by setting

$$
V_{p_{\theta}(u, \ell)}=\sum_{k=1}^{|u|} \xi^{\pi^{k}(u)}\left(h_{\pi^{k}(u)}\right)+\xi^{u}(\ell)
$$

for every $u \in \mathcal{T}$ and $\ell \in\left[0, h_{u}\right]$. Finally, with every leaf $a$ of $\tilde{\theta}$ we associate a stopped path $\mathrm{w}^{(a)}$ with lifetime $d_{\theta}(0, a)$ : For every $t \in\left[0, d_{\theta}(0, a)\right], \mathrm{w}^{(a)}(t)=V_{r(a, t)}$ where $r(a, t)$ is the unique element of $\llbracket 0, a \rrbracket$ such that $d_{\theta}(0, r(a, t))=t$.

For every integer $p \geqslant 1$, denote by $\mathbf{A}_{p}$ the set of all binary trees with $p$ leaves, and by $\mathbb{T}_{p}$ the corresponding set of marked trees. The uniform measure $\Lambda_{p}$ on $\mathbb{T}_{p}$ is defined by

$$
\int_{\mathbb{T}_{p}} \Lambda_{p}(\mathrm{~d} \theta) F(\theta)=\sum_{\mathcal{T} \in \mathbf{A}_{p}} \int \prod_{v \in \mathcal{T}} \mathrm{d} h_{v} F\left(\mathcal{T},\left(h_{v}\right)_{v \in \mathcal{T}}\right) .
$$

With this notation, Proposition IV.2 of [24] states that, for every integer $p \geqslant 1$ and every symmetric nonnegative measurable function $F$ on $\mathcal{W}^{p}$,

$$
\mathbb{N}_{x}\left(\int_{] 0, \sigma[p} \mathrm{d} s_{1} \ldots \mathrm{d} s_{p} F\left(W_{s_{1}}, \ldots, W_{s_{p}}\right)\right)=2^{p-1} p ! \int \Lambda_{p}(\mathrm{~d} \theta) Q_{x}^{\theta}\left[F\left(\left(\mathrm{w}^{(a)}\right)_{a \in L(\theta)}\right)\right] .
$$

We will need a stronger result concerning the case where the function $F$ also depends on the range $\mathcal{R}$ of the Brownian snake. To state this result, denote by $\mathcal{K}$ the space of all compact subsets of $\mathbb{R}^{d}$, which is equipped with the Hausdorff 
metric and the associated Borel $\sigma$-field. Suppose that under the probability measure $Q_{x}^{\theta}$ (for each choice of $\theta$ in $\mathbb{T}$ ), in addition to the process $\left(V_{a}, a \in \tilde{\theta}\right)$, we are also given an independent Poisson point measure on $\tilde{\theta} \times \Omega$, denoted by

$$
\sum_{i \in I} \delta_{\left(a_{i}, \omega_{i}\right)}
$$

with intensity $4 \mathcal{L}_{\theta}(\mathrm{d} a) \otimes \mathbb{N}_{0}(\mathrm{~d} \omega)$.

Theorem 2.2. For every nonnegative measurable function $F$ on $\mathcal{W}^{p} \times \mathcal{K} \times \mathbb{R}_{+}$, which is symmetric in the first $p$ variables, we have

$$
\begin{aligned}
& \mathbb{N}_{x}\left(\int_{] 0, \sigma\left[^{p}\right.} \mathrm{d} s_{1} \cdots \mathrm{d} s_{p} F\left(W_{s_{1}}, \ldots, W_{s_{p}}, \mathcal{R}, \sigma\right)\right) \\
& \quad=2^{p-1} p ! \int \Lambda_{p}(\mathrm{~d} \theta) Q_{x}^{\theta}\left[F\left(\left(\mathrm{w}^{(a)}\right)_{a \in L(\theta)}, \mathrm{cl}\left(\bigcup_{i \in I}\left(V_{a_{i}}+\mathcal{R}\left(\omega_{i}\right)\right)\right), \sum_{i \in I} \sigma\left(\omega_{i}\right)\right)\right],
\end{aligned}
$$

where $\operatorname{cl}(A)$ denotes the closure of the set $A$.

Remark. It is immediate to see that

$$
\operatorname{cl}\left(\bigcup_{i \in I}\left(V_{a_{i}}+\mathcal{R}\left(\omega_{i}\right)\right)\right)=\left(\bigcup_{a \in L(\theta)} \mathrm{w}^{(a)}\left[0, \zeta_{\left(\mathrm{w}^{(a)}\right)}\right]\right) \cup\left(\bigcup_{i \in I}\left(V_{a_{i}}+\mathcal{R}\left(\omega_{i}\right)\right)\right), \quad Q_{x}^{\theta} \text { a.e. }
$$

Proof. Consider first the case $p=1$. Let $F_{1}$ be a nonnegative measurable function on $\mathcal{W}$, and let $F_{2}$ and $F_{3}$ be two nonnegative measurable functions on $\Omega$. By applying the Markov property under $\mathbb{N}_{x}$ at time $s$, then using the timereversal invariance of $\mathbb{N}_{x}$ (which is easy from the analogous property for the Ito measure $n(\mathrm{~d} e)$ ), and finally using the Markov property at time $s$ once again, we get

$$
\begin{aligned}
& \mathbb{N}_{x}\left(\int_{0}^{\sigma} \mathrm{d} s F_{1}\left(W_{s}\right) F_{2}\left(\left(W_{(s-r)^{+}}\right)_{r \geqslant 0}\right) F_{3}\left(\left(W_{s+r}\right)_{r} \geqslant 0\right)\right) \\
& \quad=\mathbb{N}_{x}\left(\int_{0}^{\sigma} \mathrm{d} s F_{1}\left(W_{s}\right) F_{2}\left(\left(W_{(s-r)^{+}}\right)_{r} \geqslant 0\right) \mathbb{E}_{W_{s}}\left[F_{3}\left(\left(W_{r \wedge \sigma}\right)_{r \geqslant 0}\right)\right]\right) \\
& =\mathbb{N}_{x}\left(\int_{0}^{\sigma} \mathrm{d} s F_{1}\left(W_{s}\right) F_{2}\left(\left(W_{s+r}\right)_{r \geqslant 0}\right) \mathbb{E}_{W_{s}}\left[F_{3}\left(\left(W_{r \wedge \sigma}\right)_{r \geqslant 0}\right)\right]\right) \\
& =\mathbb{N}_{x}\left(\int_{0}^{\sigma} \mathrm{d} s F_{1}\left(W_{s}\right) \mathbb{E}_{W_{s}}\left[F_{2}\left(\left(W_{r \wedge \sigma}\right)_{r \geqslant 0}\right)\right] \mathbb{E}_{W_{s}}\left[F_{3}\left(\left(W_{r \wedge \sigma}\right)_{r \geqslant 0}\right)\right]\right) .
\end{aligned}
$$

We then use the case $p=1$ of (1) to see that the last quantity is equal to

$$
\int_{0}^{\infty} \mathrm{d} t \int P_{x}^{t}(\mathrm{dw}) F_{1}(\mathrm{w}) \mathbb{E}_{\mathrm{w}}\left[F_{2}\left(\left(W_{r \wedge \sigma}\right)_{r \geqslant 0}\right)\right] \mathbb{E}_{\mathrm{w}}\left[F_{3}\left(\left(W_{r \wedge \sigma}\right)_{r \geqslant 0}\right)\right],
$$

where $P_{x}^{t}$ denotes the law of Brownian motion started at $x$ and stopped at time $t$ (this law is viewed as a probability measure on $\mathcal{W})$. Now if we specialize to the case where $F_{2}$ is a function of the form $F_{2}(\omega)=G_{2}\left(\left\{\widehat{W}_{s}(\omega): s \geqslant 0\right\}, \sigma\right)$, an immediate application of Lemma V.2 in [24] shows that

$$
\mathbb{E}_{\mathrm{w}}\left[F_{2}\left(\left(W_{r \wedge \sigma}\right)_{r \geqslant 0}\right)\right]=E\left[G_{2}\left(\operatorname{cl}\left(\bigcup_{j \in J}\left(\mathrm{w}\left(t_{j}\right)+\mathcal{R}\left(\omega_{j}\right)\right)\right), \sum_{j \in J} \sigma\left(\omega_{j}\right)\right)\right],
$$


where $\sum_{j \in J} \delta_{\left(t_{j}, \omega_{j}\right)}$ is a Poisson point measure on $\left[0, \zeta_{(\mathrm{w})}\right] \times \Omega$ with intensity $2 \mathrm{~d} t \mathbb{N}_{0}(\mathrm{~d} \omega)$. Applying the same observation to $F_{3}$, we easily get the case $p=1$ of the theorem.

The general case can be derived along similar lines by using Theorem 3 in [22]. Roughly speaking, the case $p=1$ amounts to combining Bismut's decomposition of the Brownian excursion (Lemma 1 in [22]) with the spatial displacements of the Brownian snake. For general $p$, the second assertion of Theorem 3 in [22] provides the analogue of Bismut's decomposition, which when combined with spatial displacements leads to the statement of Theorem 2.2. Details are left to the reader.

\subsection{The re-rooting theorem}

In this subsection, we state and prove an important invariance property of the Brownian snake under $\mathbb{N}_{0}$, which plays a major role in Section 3 below. We first need to introduce some notation. For every $s, r \in[0, \sigma]$, we set

$$
s \oplus r= \begin{cases}s+r & \text { if } s+r \leqslant \sigma \\ s+r-\sigma & \text { if } s+r>\sigma .\end{cases}
$$

We also use the following convenient notation for closed intervals: If $u, v \in \mathbb{R},[u, v]=[v, u]=[u \wedge v, u \vee v]$

Let $s \in\left[0, \sigma\left[\right.\right.$. In order to define the re-rooted snake $W^{[s]}$, we first set

$$
\zeta_{r}^{[s]}=\zeta_{s}+\zeta_{s \oplus r}-2 \inf _{u \in[s, s \oplus r]} \zeta_{u},
$$

if $r \in[0, \sigma]$, and $\zeta_{r}^{[s]}=0$ if $r>\sigma$. We also want to define the stopped paths $W_{r}^{[s]}$, in such a way that

$$
\widehat{W}_{r}^{[s]}=\widehat{W}_{s \oplus r}-\widehat{W}_{s},
$$

if $r \in[0, \sigma]$, and $\widehat{W}_{r}^{[s]}=0$ if $r>\sigma$. To this end, we may notice that $\widehat{W}^{[s]}$ satisfies the property

$$
\widehat{W}_{r}^{[s]}=\widehat{W}_{r^{\prime}}^{[s]} \quad \text { if } \zeta_{r}^{[s]}=\zeta_{r^{\prime}}^{[s]}=\inf _{u \in\left[r, r^{\prime}\right]} \zeta_{u}^{[s]}
$$

and so in the terminology of [26], $\left(W_{r}^{[s]}\right)_{0 \leqslant r \leqslant \sigma}$ is uniquely determined as the snake whose tour is $\left(\zeta_{r}^{[s]}, \widehat{W}_{r}^{[s]}\right)_{0 \leqslant r \leqslant \sigma}$ (see the homeomorphism theorem of [26]). We have the explicit formula, for $r \geqslant 0$, and $0 \leqslant t \leqslant \zeta_{r}^{[s]}$,

$$
W_{r}^{[s]}(t)=\widehat{W}_{\sup \left\{u \leqslant r: \zeta_{u}^{[s]}=t\right\}}^{[s]} .
$$

As explained in the introduction, $\left(\zeta_{r}^{[s]}\right)_{r \geqslant 0}$ codes the same $\mathbb{R}$-tree as the one coded by $\left(\zeta_{r}\right)_{r} \geqslant 0$, but with a new root which is the vertex originally labeled by $s$, and $W_{r}^{[s]}$ gives the spatial displacements along the line segment from the (new) root to the vertex coded by $r$ (in the coding given by $\zeta^{[s]}$ ).

Theorem 2.3. For every nonnegative measurable function $F$ on $\mathbb{R}_{+} \times \Omega$,

$$
\mathbb{N}_{0}\left(\int_{0}^{\sigma} \mathrm{d} s F\left(s, W^{[s]}\right)\right)=\mathbb{N}_{0}\left(\int_{0}^{\sigma} \mathrm{d} s F(s, W)\right) .
$$

Remark. For every $s \in\left[0, \sigma\right.$, the duration of the re-rooted snake excursion $W^{[s]}$ is the same as that of the original one. Using this simple observation, and replacing $F$ by $\mathbf{1}_{\{1-\varepsilon<\sigma \leqslant 1\}} F$, we can easily get a version of Theorem 2.3 for the normalized Brownian snake excursion. Precisely, the formula of Theorem 2.3 still holds if $\mathbb{N}_{0}$ is replaced by $\mathbb{N}_{0}^{(1)}$ (or by $\mathbb{N}_{0}^{(r)}$ for any $r>0$ ). Via a continuity argument, it follows that, for every $s \in[0,1[$, and every nonnegative measurable function $G$ on $\Omega$,

$$
\mathbb{N}_{0}^{(1)}\left(G\left(W^{[s]}\right)\right)=\mathbb{N}_{0}^{(1)}(G(W)) .
$$

The identity (3) appears as Proposition 4.9 of [27], which is proved via discrete approximations. Note that conversely, it would be easy to derive Theorem 2.3 from (3). We have chosen to give an independent proof of Theorem 2.3 because this result plays a major role in the present work, and also because the proof below fits in better with our general strategy, which is to deal first with unnormalized excursion measures before conditioning with respect to the duration. 
Proof. By (2), $W^{[s]}$ can be written $\mathbb{N}_{0}$ a.e. as $\Phi\left(\zeta^{[s]}, \widehat{W}^{[s]}\right)$, where the deterministic function $\Phi$ does not depend on $s$. Also note that when $s=0, W=W^{[0]}=\Phi(\zeta, \widehat{W}), \mathbb{N}_{0}$ a.e. In view of these considerations, it will be sufficient to treat the case when

$$
F(s, W)=F_{1}(s, \zeta) F_{2}(s, \widehat{W})
$$

where $F_{1}$ and $F_{2}$ are nonnegative measurable functions defined respectively on $\mathbb{R}_{+} \times C\left(\mathbb{R}_{+}, \mathbb{R}_{+}\right)$and on $\mathbb{R}_{+} \times$ $C\left(\mathbb{R}_{+}, \mathbb{R}^{d}\right)$. We first deal with the special case $F_{2}=1$.

For $s \in[0, \sigma[$ and $r \geqslant 0$, set

$$
\begin{aligned}
& \zeta_{r}^{1, s}=\zeta_{(s-r)^{+}}-\zeta_{s}, \\
& \zeta_{r}^{2, s}=\zeta_{s+r}-\zeta_{s} .
\end{aligned}
$$

Let $G$ be a nonnegative measurable function on $\mathbb{R}_{+} \times C\left(\mathbb{R}_{+}, \mathbb{R}\right) \times \mathbb{R}_{+} \times C\left(\mathbb{R}_{+}, \mathbb{R}\right)$. From the Bismut decomposition of the Brownian excursion (see e.g. Lemma 1 in [22]), we have

$$
\mathbb{N}_{0}\left(\int_{0}^{\sigma} \mathrm{d} s G\left(s,\left(\zeta_{r}^{1, s}\right)_{r \geqslant 0}, \sigma-s,\left(\zeta_{r}^{2, s}\right)_{r \geqslant 0}\right)\right)=\int_{0}^{\infty} \mathrm{d} a E\left[G\left(T_{a},\left(B_{r \wedge T_{a}}\right)_{r \geqslant 0}, T_{a}^{\prime},\left(B_{r \wedge T_{a}^{\prime}}^{\prime}\right)_{r \geqslant 0}\right)\right],
$$

where $B$ and $B^{\prime}$ are two independent linear Brownian motions started at 0 , and

$$
T_{a}=\inf \left\{r \geqslant 0: B_{r}=-a\right\}, \quad T_{a}^{\prime}=\inf \left\{r \geqslant 0: B_{r}^{\prime}=-a\right\} .
$$

Now observe that

$$
\begin{aligned}
& \zeta_{r}^{[s]}=\zeta_{r}^{2, s}-2 \inf _{0 \leqslant u \leqslant r} \zeta_{u}^{2, s}, \quad \text { if } 0 \leqslant r \leqslant \sigma-s, \\
& \zeta_{\sigma-r}^{[s]}=\zeta_{r}^{1, s}-2 \inf _{0 \leqslant u \leqslant r} \zeta_{u}^{1, s}, \quad \text { if } 0 \leqslant r \leqslant s,
\end{aligned}
$$

and note that $R_{t}:=B_{t}-2 \inf _{r \leqslant t} B_{r}$ and $R_{r}^{\prime}:=B_{t}^{\prime}-2 \inf _{r \leqslant t} B_{r}^{\prime}$ are two independent three-dimensional Bessel processes, for which

$$
\begin{aligned}
& L_{a}:=\sup \left\{t \geqslant 0: R_{t} \leqslant a\right\}=T_{a}, \\
& L_{a}^{\prime}:=\sup \left\{t \geqslant 0: R_{t}^{\prime} \leqslant a\right\}=T_{a}^{\prime} .
\end{aligned}
$$

(This is Pitman's theorem, see e.g. [28], Theorem VI.3.5.) It follows that

$$
\begin{aligned}
\mathbb{N}_{0}\left(\int_{0}^{\sigma} \mathrm{d} s G\left(\sigma-s,\left(\zeta_{r \wedge(\sigma-s)}^{[s]}\right)_{r \geqslant 0}, s,\left(\zeta_{\sigma-(r \wedge s)}^{[s]}\right)_{r \geqslant 0}\right)\right) & =\int_{0}^{\infty} \mathrm{d} a E\left[G\left(L_{a}^{\prime},\left(R_{r \wedge L_{a}^{\prime}}^{\prime}\right)_{r \geqslant 0}, L_{a},\left(R_{r \wedge L_{a}}\right)_{r \geqslant 0}\right)\right) \\
& =\mathbb{N}_{0}\left(\int_{0}^{\sigma} \mathrm{d} s G\left(s,\left(\zeta_{r \wedge s}\right)_{r \geqslant 0}, \sigma-s,\left(\zeta_{(\sigma-r) \vee s}\right)_{r \geqslant 0}\right)\right)
\end{aligned}
$$

where the last equality is again a consequence of the Bismut decomposition, together with the Williams reversal theorem ([28], Corollary XII.4.4). Changing $s$ into $\sigma-s$ in the last integral gives the desired result when $F_{2}=1$.

Let us consider the general case. For simplicity we take $d=1$, but the argument can obviously be extended. From the definition of the Brownian snake, we have

$$
\mathbb{N}_{0}\left(\int_{0}^{\sigma} \mathrm{d} s F_{1}(s, \zeta) F_{2}(s, \widehat{W})\right)=\mathbb{N}_{0}\left(\int_{0}^{\sigma} \mathrm{d} s F_{1}(s, \zeta) \Theta_{0}^{\zeta}\left[F_{2}(s, \widehat{W})\right]\right)
$$

and $\widehat{W}$ is under $\Theta_{0}^{\zeta}$ a centered Gaussian process with covariance

$$
\operatorname{cov}_{\Theta_{0}^{\zeta}}\left(\widehat{W}_{s}, \widehat{W}_{s^{\prime}}\right)=\inf _{r \in\left[s, s^{\prime}\right]} \zeta_{r} .
$$


We have in particular

$$
\mathbb{N}_{0}\left(\int_{0}^{\sigma} \mathrm{d} s F_{1}\left(s, \zeta^{[s]}\right) F_{2}\left(s, \widehat{W}^{[s]}\right)\right)=\mathbb{N}_{0}\left(\int_{0}^{\sigma} \mathrm{d} s F_{1}\left(s, \zeta^{[s]}\right) \Theta_{0}^{\zeta}\left[F_{2}\left(s,\left(\widehat{W}_{s \oplus r}-\widehat{W}_{s}\right)_{r \geqslant 0}\right)\right]\right) .
$$

Now note that $\left(\widehat{W}_{s \oplus r}-\widehat{W}_{s}\right)_{r \geqslant 0}$ is under $\Theta_{0}^{\zeta}$ a Gaussian process with covariance

$$
\operatorname{cov}\left(\widehat{W}_{s \oplus r}-\widehat{W}_{s}, \widehat{W}_{s \oplus r^{\prime}}-\widehat{W}_{s}\right)=\inf _{\left[s \oplus r, s \oplus r^{\prime}\right]} \zeta_{u}-\inf _{[s \oplus r, s]} \zeta_{u}-\inf _{\left[s \oplus r^{\prime}, s\right]} \zeta_{u}+\zeta_{s}=\inf _{\left[r, r^{\prime}\right]} \zeta_{u}^{[s]},
$$

where the last equality follows from an elementary verification. Hence,

$$
\Theta_{0}^{\zeta}\left[F_{2}\left(s,\left(\widehat{W}_{s \oplus r}-\widehat{W}_{s}\right)_{r \geqslant 0}\right)\right]=\Theta_{0}^{\zeta^{[s]}}\left[F_{2}(s, \widehat{W})\right],
$$

and, using the first part of the proof,

$$
\begin{aligned}
\mathbb{N}_{0}\left(\int_{0}^{\sigma} \mathrm{d} s F_{1}\left(s, \zeta^{[s]}\right) F_{2}\left(s, \widehat{W}^{[s]}\right)\right) & =\mathbb{N}_{0}\left(\int_{0}^{\sigma} \mathrm{d} s F_{1}\left(s, \zeta^{[s]}\right) \Theta_{0}^{\zeta s]}\left[F_{2}(s, \widehat{W})\right]\right) \\
& =\mathbb{N}_{0}\left(\int_{0}^{\sigma} \mathrm{d} s F_{1}(s, \zeta) \Theta_{0}^{\zeta}\left[F_{2}(s, \widehat{W})\right]\right) \\
& =\mathbb{N}_{0}\left(\int_{0}^{\sigma} \mathrm{d} s F_{1}(s, \zeta) F_{2}(s, \widehat{W})\right) .
\end{aligned}
$$

This completes the proof.

\subsection{The special Markov property}

Let $D$ be a domain in $\mathbb{R}^{d}$, and fix a point $x \in D$. For every $\mathrm{w} \in \mathcal{W}$, we set

$$
\tau(\mathrm{w}):=\inf \{t \geqslant 0: \mathrm{w}(t) \notin D\}
$$

where $\inf \varnothing=+\infty$ as usual. The random set

$$
\left\{s \geqslant 0: \tau\left(W_{s}\right)<\zeta_{s}\right\}
$$

is open $\mathbb{N}_{x}$ a.e., and can thus be written as a disjoint union of open intervals $] a_{i}, b_{i}$ [, $i \in I$. It is easy to verify that $\mathbb{N}_{x}$ a.e. for every $i \in I$ and every $s \in] a_{i}, b_{i}[$,

$$
\tau\left(W_{s}\right)=\tau\left(W_{a_{i}}\right)=\tau\left(W_{b_{i}}\right)=\zeta_{a_{i}}=\zeta_{b_{i}}
$$

and moreover the paths $W_{s}, s \in\left[a_{i}, b_{i}\right]$, coincide up to their exit time from $D$.

For every $i \in I$, we define a random element $W^{(i)}$ of $\Omega$ by setting for every $s \geqslant 0$

$$
W_{s}^{(i)}(t)=W_{\left(a_{i}+s\right) \wedge b_{i}}\left(\zeta_{a_{i}}+t\right), \quad \text { for } 0 \leqslant t \leqslant \zeta_{\left(W_{s}^{(i)}\right)}:=\zeta_{\left(a_{i}+s\right) \wedge b_{i}}-\zeta_{a_{i}} .
$$

Informally, the $W^{(i)}$ 's represent the excursions of the Brownian snake outside $D$ (the word "outside" is a bit misleading since these excursions may come back into $D$ even though they start from the boundary of $D$ ).

Finally, we also need a process that contains the information given by the Brownian snake paths before they exit $D$. We set $\widetilde{W}_{s}^{D}=W_{\eta_{s}^{D}}$, where for every $s \geqslant 0$,

$$
\eta_{s}^{D}:=\inf \left\{r \geqslant 0: \int_{0}^{r} \mathrm{~d} u \mathbf{1}_{\left\{\tau\left(W_{u}\right) \geqslant \zeta_{u}\right\}}>s\right\} .
$$

The $\sigma$-field $\mathcal{E}^{D}$ is by definition generated by the process $\widetilde{W}^{D}$ and by the class of $\mathbb{N}_{x}$-negligible subsets of $\Omega$ (the point $x$ is fixed throughout this subsection). The following statement is proved in [23] (Proposition 2.3 and Theorem 2.4). 
Theorem 2.4. There exists a random finite measure denoted by $\mathcal{Z}^{D}$, which is $\mathcal{E}^{D}$-measurable and $\mathbb{N}_{x}$ a.e. supported on $\partial D$, such that the following holds. Under $\mathbb{N}_{x}$, conditionally on $\mathcal{E}^{D}$, the point measure

$$
\mathcal{N}:=\sum_{i \in I} \delta_{\left(W^{(i)}\right)}
$$

is Poisson with intensity $\int_{\partial D} \mathcal{Z}^{D}(\mathrm{~d} y) \mathbb{N}_{y}(\cdot)$.

We will apply this theorem to the case $d=1, x=0$ and $D=] c, \infty[$ for some $c<0$. In that case, the measure $\mathcal{Z}^{D}$ is a random multiple of the Dirac measure at $c: \mathcal{Z}^{D}=L^{c} \delta_{c}$ for some nonnegative random variable $L^{c}$. From Lemma 2.1(i) and Theorem 2.4, it is easy to verify that $\left.\left.\left\{L^{c}>0\right\}=\{\mathcal{R} \cap]-\infty, c\right] \neq \varnothing\right\}=\{\mathcal{R} \cap]-\infty, c[\neq \varnothing\}$, $\mathbb{N}_{0}$ a.e. Moreover, as a simple consequence of the special Markov property, the process $\left(L^{-r}\right)_{r>0}$ is a nonnegative martingale under $\mathbb{N}_{0}$ (it is indeed a critical continuous-state branching process). In particular the variable

$$
L^{*, r}:=\sup _{c \in \mathbb{Q} \cap]-\infty, r]} L^{c}
$$

is finite $\mathbb{N}_{0}$ a.e., for every $r<0$.

\subsection{Uniqueness of the minimum}

From now on we assume that $d=1$. In this subsection, we consider the Brownian snake under its excursion measure $\mathbb{N}_{0}$. We use the notation

$$
\underline{W}=\inf _{s \geqslant 0} \widehat{W}_{s} .
$$

Note that the law of $\underline{W}$ under $\mathbb{N}_{0}$ is given by Lemma 2.1(i) and an obvious translation argument.

Proposition 2.5. There exists $\mathbb{N}_{0}$ a.e. a unique instant $\left.s_{*} \in\right] 0, \sigma\left[\right.$ such that $\widehat{W}_{s_{*}}=\underline{W}$.

This result already appears as Lemma 16 in [27], where its proof is attributed to T. Duquesne. We provide a short proof for the sake of completeness and also because this result plays a major role throughout this work.

\section{Proof. Set}

$$
\lambda:=\inf \left\{s \geqslant 0: \widehat{W}_{s}=\underline{W}\right\}, \quad \rho=\sup \left\{s \geqslant 0: \widehat{W}_{s}=\underline{W}\right\}
$$

so that $0<\lambda \leqslant \rho<\sigma$. We have to prove that $\lambda=\rho$. To this end we fix $\delta>0$ and we verify that $\mathbb{N}_{0}(\rho-\lambda>\delta)=0$.

Fix two rational numbers $q<0$ and $\varepsilon>0$. We first get an upper bound on the quantity

$$
\mathbb{N}_{0}(q-\varepsilon \leqslant \underline{W}<q, \rho-\lambda>\delta) .
$$

Denote by $\left(W^{(i)}\right)_{i \in I}$ the excursions of the Brownian snake outside $] q, \infty[$, and by $\mathcal{N}$ the corresponding point measure, as in the previous subsection. Since the law of $\underline{W}$ under $\mathbb{N}_{0}$ has no atoms, the numbers $\underline{W}^{(i)}, i \in I$, are distinct $\mathbb{N}_{0}$ a.e. Therefore, on the event $\{\underline{W}<q\}$, the whole interval $[\rho, \lambda]$ must be contained in a single excursion interval below level $q$. Hence,

$$
\mathbb{N}_{0}(q-\varepsilon \leqslant \underline{W}<q, \rho-\lambda>\delta) \leqslant \mathbb{N}_{0}\left(\left\{\forall i \in I: \underline{W}^{(i)} \geqslant q-\varepsilon\right\} \cap\left\{\exists i \in I: \sigma\left(W^{(i)}\right)>\delta\right\}\right) .
$$

Introduce the events $A_{\varepsilon}:=\{\underline{W}<q-\varepsilon\}$ and $B_{\varepsilon, \delta}=\{\underline{W} \geqslant q-\varepsilon, \sigma>\delta\}$. We get

$$
\mathbb{N}_{0}(q-\varepsilon \leqslant \underline{W}<q, \rho-\lambda>\delta) \leqslant \mathbb{N}_{0}\left(\mathcal{N}\left(A_{\varepsilon}\right)=0, \mathcal{N}\left(B_{\varepsilon, \delta}\right) \geqslant 1\right) .
$$

From the special Markov property (and the remarks of the end of Subsection 2.3), we know that conditionally on $L^{q}$, $\mathcal{N}$ is a Poisson point measure with intensity $L^{q} \mathbb{N}_{q}$. Since the sets $A_{\varepsilon}$ and $B_{\varepsilon, \delta}$ are disjoint, independence properties of Poisson point measures give

$$
\begin{aligned}
\mathbb{N}_{0}(q-\varepsilon \leqslant \underline{W}<q, \rho-\lambda>\delta) & \leqslant \mathbb{N}_{0}\left(\mathbf{1}_{\left\{\mathcal{N}\left(A_{\varepsilon}\right)=0\right\}}\left(1-\exp \left(-L^{q} \mathbb{N}_{q}\left(B_{\varepsilon, \delta}\right)\right)\right)\right) \\
& =\mathbb{N}_{0}\left(\mathbf{1}_{\{q-\varepsilon \leqslant \underline{W}<q\}}\left(1-\exp \left(-L^{q} \mathbb{N}_{q}\left(B_{\varepsilon, \delta}\right)\right)\right)\right) \\
& \leqslant \mathbb{N}_{0}\left(\mathbf{1}_{\{q-\varepsilon \leqslant \underline{W}<q\}}\left(1-\exp \left(-c(\varepsilon, \delta) L^{*, q}\right)\right)\right)
\end{aligned}
$$


where $c(\varepsilon, \delta)=\mathbb{N}_{q}\left(B_{\varepsilon, \delta}\right)$ does not depend on $q$ by an obvious translation argument.

We can apply the preceding bound with $q$ replaced by $q-\varepsilon, q-2 \varepsilon$, etc. By summing the resulting estimates we get

$$
\mathbb{N}_{0}(\underline{W}<q, \rho-\lambda>\delta) \leqslant \mathbb{N}_{0}\left(\mathbf{1}_{\{\underline{W}<q\}}\left(1-\exp \left(-c(\varepsilon, \delta) L^{*, q}\right)\right)\right) .
$$

Clearly $c(\varepsilon, \delta)$ tends to 0 as $\varepsilon \rightarrow 0$, and dominated convergence gives $\mathbb{N}_{0}(\underline{W}<q, \rho-\lambda>\delta)=0$. This completes the proof since $q$ was arbitrary.

\subsection{Bessel processes}

Throughout this work, $\left(\xi_{t}\right)_{t \geqslant 0}$ will stand for a linear Brownian motion started at $x$ under the probability measure $P_{x}$. The notation $\xi[0, t]$ will stand for the range of $\xi$ over the time interval $[0, t]$. For every $\delta>0,\left(R_{t}\right)_{t} \geqslant 0$ will denote a Bessel process of dimension $\delta$ started at $x$ under the probability measure $P_{x}^{(\delta)}$. We will use repeatedly the following simple facts. First, if $\lambda>0$, the process $R_{t}^{(\lambda)}:=\lambda^{-1} R_{\lambda^{2} t}$ is under $P_{x}^{(\delta)}$ a Bessel process of dimension $\delta$ started at $x / \lambda$. Secondly, if $0 \leqslant x \leqslant x^{\prime}$ and $t \geqslant 0$, the law of $R_{t}$ under $P_{x}^{(\delta)}$ is stochastically bounded by the law of $R_{t}$ under $P_{x^{\prime}}^{(\delta)}$. The latter fact follows from standard comparison theorems applied to squared Bessel processes.

Absolute continuity relations between Bessel processes, which are consequences of the Girsanov theorem, were first observed by Yor [31]. We state a special case of these relations, which will play an important role in this work. This special case appears in Exercise XI.1.22 of [28].

Proposition 2.6. Let $t>0$ and let $F$ be a nonnegative measurable function on $C([0, t], \mathbb{R})$. Then, for every $x>0$ and $\lambda>0$,

$$
E_{x}\left[\mathbf{1}_{\{\xi[0, t] \subset] 0, \infty[\}} \exp \left(-\frac{\lambda^{2}}{2} \int_{0}^{t} \frac{\mathrm{d} r}{\xi_{r}^{2}}\right) F\left(\left(\xi_{r}\right)_{0 \leqslant r \leqslant t}\right)\right]=x^{\nu+1 / 2} E_{x}^{(2+2 v)}\left[\left(R_{t}\right)^{-v-1 / 2} F\left(\left(R_{r}\right)_{0 \leqslant r \leqslant t}\right)\right],
$$

where $v=\sqrt{\lambda^{2}+\frac{1}{4}}$.

We shall be concerned by the case when $\lambda^{2} / 2=6$, and then $2+2 v=9$ and $v+1 / 2=4$. Taking $F=1$ in that case, we see that

$$
x^{4} E_{x}^{(9)}\left[R_{t}^{-4}\right] \leqslant 1 .
$$

\section{Conditioning and re-rooting of trees}

This section contains the proof of Theorems 1.1 and 1.2 which were stated in the introduction. Both will be derived as consequences of Theorem 3.1 below. Recall the notation $s_{*}$ for the unique time of the minimum of $\widehat{W}$ under $\mathbb{N}_{0}$, and $W^{[s]}$ for the snake re-rooted at $s$.

Theorem 3.1. Let $\varphi: \mathbb{R}_{+} \rightarrow \mathbb{R}_{+}$be a continuous function such that $\varphi(s) \leqslant C(1 \wedge s)$ for some finite constant $C$. Let $F: \Omega \rightarrow \mathbb{R}_{+}$be a bounded continuous function. Then,

$$
\lim _{\varepsilon \rightarrow 0} \varepsilon^{-4} \mathbb{N}_{0}\left(\sigma \varphi(\sigma) F(W) \mathbf{1}_{\{\underline{W}>-\varepsilon\}}\right)=\frac{2}{21} \mathbb{N}_{0}\left(\varphi(\sigma) F\left(W^{\left[s_{*}\right]}\right)\right) .
$$

The proof of Theorem 3.1 occupies most of the remainder of this section. This proof will depend on a series of lemmas. To motivate these lemmas, we first observe that, from the re-rooting identity Theorem 2.3 , we have

$$
\begin{aligned}
\mathbb{N}_{0}\left(\sigma \varphi(\sigma) F(W) \mathbf{1}_{\{\underline{W}>-\varepsilon\}}\right) & =\mathbb{N}_{0}\left(\varphi(\sigma) \int_{0}^{\sigma} \mathrm{d} s F\left(W^{[s]}\right) \mathbf{1}_{\left\{\underline{W}^{[s]}>-\varepsilon\right\}}\right) \\
& =\mathbb{N}_{0}\left(\varphi(\sigma) \int_{0}^{\sigma} \mathrm{d} s F\left(W^{[s]}\right) \mathbf{1}_{\left\{\widehat{W}_{s}<\underline{W}+\varepsilon\right\}}\right),
\end{aligned}
$$


since $\underline{W}^{[s]}=\underline{W}-\widehat{W}_{s}$ by construction. The fact that the minimum of $\widehat{W}$ is attained at a unique time implies that

$$
\sup \left\{\left|s-s_{*}\right|: \widehat{W}_{s}<\underline{W}+\varepsilon\right\} \underset{\varepsilon \rightarrow 0}{\longrightarrow} 0, \quad \mathbb{N}_{0} \text { a.e. }
$$

and it easily follows that

$$
\sup \left\{\left|F\left(W^{[s]}\right)-F\left(W^{\left[s_{*}\right]}\right)\right|: \widehat{W}_{s}<\underline{W}+\varepsilon\right\} \underset{\varepsilon \rightarrow 0}{\longrightarrow} 0, \quad \mathbb{N}_{0} \text { a.e. }
$$

Coming back to (5), this suggests to study the behavior of

$$
\int_{0}^{\sigma} \mathrm{d} s \mathbf{1}_{\left\{\widehat{W}_{s}<\underline{W}+\varepsilon\right\}}
$$

as $\varepsilon \rightarrow 0$. This motivates the next two lemmas.

\section{Lemma 3.2. We have}

$$
\lim _{\delta \downarrow 0} \sup _{0<\varepsilon<1}\left(\varepsilon^{-4} \mathbb{N}_{0}\left(\left(1-\mathrm{e}^{-\sigma}\right) \int_{0}^{\sigma} \mathrm{d} s \mathbf{1}_{\left\{\widehat{W}_{s}<\underline{W}+\varepsilon, \zeta_{s}<\delta\right\}}\right)\right)=0 .
$$

Proof. From Theorem 2.3 and the fact that $\zeta_{s}=\zeta_{\sigma-s}^{[s]}$ for every $s \in[0, \sigma]$, we have

$$
\begin{aligned}
\mathbb{N}_{0}\left(\left(1-\mathrm{e}^{-\sigma}\right) \int_{0}^{\sigma} \mathrm{d} s \mathbf{1}_{\left\{\widehat{W}_{s}<\underline{W}+\varepsilon, \zeta_{s}<\delta\right\}}\right) & =\mathbb{N}_{0}\left(\left(1-\mathrm{e}^{-\sigma}\right) \int_{0}^{\sigma} \mathrm{d} s \mathbf{1}_{\left\{\underline{W}^{[s]}>-\varepsilon, \zeta_{\sigma-s}^{[s]}<\delta\right\}}\right) \\
& =\mathbb{N}_{0}\left(\left(1-\mathrm{e}^{-\sigma}\right) \int_{0}^{\sigma} \mathrm{d} s \mathbf{1}_{\left\{\underline{W}>-\varepsilon, \zeta_{\sigma-s}<\delta\right\}}\right) \\
& =\mathbb{N}_{0}\left(\left(1-\mathrm{e}^{-\sigma}\right) \mathbf{1}_{\{\underline{W}>-\varepsilon\}} \int_{0}^{\sigma} \mathrm{d} s \mathbf{1}_{\left\{\zeta_{s}<\delta\right\}}\right) .
\end{aligned}
$$

Recall that $\left(\xi_{t}\right)_{t \geqslant 0}$ denotes a standard linear Brownian motion that starts from $x$ under the probability measure $P_{x}$, and write $\underline{\xi}_{t}:=\inf \left\{\xi_{r}: r \leqslant t\right\}$. From the case $p=1$ of Theorem 2.2 and Lemma 2.1(i) we get

$$
\begin{aligned}
\mathbb{N}_{0}\left(\mathbf{1}_{\{\underline{W}>-\varepsilon\}} \int_{0}^{\sigma} \mathrm{d} s \mathbf{1}_{\left\{\zeta_{s}<\delta\right\}}\right) & =\int_{0}^{\delta} \mathrm{d} a E_{0}\left[\mathbf{1}_{\left\{\underline{\xi}_{a}>-\varepsilon\right\}} \exp \left(-4 \int_{0}^{a} \mathrm{~d} t \mathbb{N}_{\xi_{t}}\left(\underline{W}_{t} \leqslant-\varepsilon\right)\right)\right] \\
& =\int_{0}^{\delta} \mathrm{d} a E_{\varepsilon}\left[\mathbf{1}_{\left\{\underline{\xi}_{a}>0\right\}} \exp \left(-6 \int_{0}^{a} \frac{\mathrm{d} t}{\xi_{t}^{2}}\right)\right] .
\end{aligned}
$$

At this point we use Proposition 2.6, which gives

$$
E_{\varepsilon}\left[\mathbf{1}_{\left\{\underline{\xi}_{a}>0\right\}} \exp \left(-6 \int_{0}^{a} \frac{\mathrm{d} t}{\xi_{t}^{2}}\right)\right]=\varepsilon^{4} E_{\varepsilon}^{(9)}\left[R_{a}^{-4}\right] .
$$

Similarly,

$$
\mathbb{N}_{0}\left(\mathbf{1}_{\{\underline{W}>-\varepsilon\}} \mathrm{e}^{-\sigma} \int_{0}^{\sigma} \mathrm{d} s \mathbf{1}_{\left\{\zeta_{s}<\delta\right\}}\right)=\int_{0}^{\delta} \mathrm{d} a E_{0}\left[\mathbf{1}_{\left\{\underline{\xi}_{a}>-\varepsilon\right\}} \exp \left(-4 \int_{0}^{a} \mathrm{~d} t \mathbb{N}_{\xi_{t}}\left(1-\mathbf{1}_{\{\underline{W}>-\varepsilon)\}} \mathrm{e}^{-\sigma}\right)\right)\right]
$$




$$
\begin{aligned}
& =\int_{0}^{\delta} \mathrm{d} a E_{\varepsilon}\left[\mathbf{1}_{\left\{\underline{\xi}_{a}>0\right\}} \exp \left(-4 \int_{0}^{a} \mathrm{~d} t \mathbb{N}_{\xi_{t}}\left(1-\mathbf{1}_{\{\underline{W}>0)\}} \mathrm{e}^{-\sigma}\right)\right)\right] \\
& =\int_{0}^{\delta} \mathrm{d} a E_{\varepsilon}\left[\mathbf{1}_{\left\{\underline{\xi}_{a}>0\right\}} \exp \left(-2^{3 / 2} \int_{0}^{a} \mathrm{~d} t\left(3 \operatorname{coth}\left(2^{1 / 4} \xi_{t}\right)^{2}-2\right)\right)\right]
\end{aligned}
$$

using Lemma 2.1(ii) in the last equality. For every $x>0$, set

$$
h(x)=-\frac{3}{2 x^{2}}+2^{-1 / 2}\left(3 \operatorname{coth}\left(2^{1 / 4} x\right)^{2}-2\right)>0 .
$$

A Taylor expansion shows that $h(x) \leqslant C x^{2}$. (Here and later, $C, C^{\prime}, C^{\prime \prime}$ denote constants whose exact value is unimportant.) Then,

$$
\begin{aligned}
\mathbb{N}_{0}\left(\mathbf{1}_{\{\underline{W}>-\varepsilon\}} \mathrm{e}^{-\sigma} \int_{0}^{\sigma} \mathrm{d} s \mathbf{1}_{\left\{\zeta_{s}<\delta\right\}}\right) & =\int_{0}^{\delta} \mathrm{d} a E_{\varepsilon}\left[\mathbf{1}_{\left\{\underline{\xi}_{a}>0\right\}} \exp \left(-6 \int_{0}^{a} \frac{\mathrm{d} t}{\xi_{t}^{2}}-4 \int_{0}^{a} \mathrm{~d} t h\left(\xi_{t}\right)\right)\right] \\
& =\int_{0}^{\delta} \mathrm{d} a \varepsilon^{4} E_{\varepsilon}^{(9)}\left[R_{a}^{-4} \exp \left(-4 \int_{0}^{a} \mathrm{~d} t h\left(R_{t}\right)\right)\right]
\end{aligned}
$$

using Proposition 2.6 as above.

By combining the preceding calculations, we arrive at

$$
\begin{aligned}
\varepsilon^{-4} \mathbb{N}_{0}\left(\mathbf{1}_{\{\underline{W}>-\varepsilon\}}\left(1-\mathrm{e}^{-\sigma}\right) \int_{0}^{\sigma} \mathrm{d} s \mathbf{1}_{\left\{\zeta_{s}<\delta\right\}}\right) & =\int_{0}^{\delta} \mathrm{d} a E_{\varepsilon}^{(9)}\left[R_{a}^{-4}\left(1-\exp \left(-4 \int_{0}^{a} \mathrm{~d} t h\left(R_{t}\right)\right)\right)\right] \\
& \leqslant 4 C \int_{0}^{\delta} \mathrm{d} a \int_{0}^{a} \mathrm{~d} t E_{\varepsilon}^{(9)}\left[R_{a}^{-4} R_{t}^{2}\right] .
\end{aligned}
$$

If $a \leqslant \delta<1 / 2$ and $0<t \leqslant a^{2}$, we can bound

$$
E_{\varepsilon}^{(9)}\left[R_{a}^{-4} R_{t}^{2}\right]=E_{\varepsilon}^{(9)}\left[R_{t}^{2} E_{R_{t}}^{(9)}\left[R_{a-t}^{-4}\right]\right] \leqslant E_{\varepsilon}^{(9)}\left[R_{t}^{2}\right] E_{0}^{(9)}\left[R_{a-t}^{-4}\right] \leqslant C^{\prime} a^{-2} .
$$

If $a^{2}<t \leqslant a$ we use a different argument: From the bound (4), we get

$$
E_{\varepsilon}^{(9)}\left[R_{a}^{-4} R_{t}^{2}\right]=E_{\varepsilon}^{(9)}\left[R_{t}^{2} E_{R_{t}}^{(9)}\left[R_{a-t}^{-4}\right]\right] \leqslant E_{\varepsilon}^{(9)}\left[R_{t}^{-2}\right] \leqslant E_{0}^{(9)}\left[R_{t}^{-2}\right]=C^{\prime \prime} t^{-1} .
$$

By substituting the bounds (10) and (11) into (9), we arrive at

$$
\varepsilon^{-4} \mathbb{N}_{0}\left(\mathbf{1}_{\{\underline{W}>-\varepsilon\}}\left(1-\mathrm{e}^{-\sigma}\right) \int_{0}^{\sigma} \mathrm{d} s \mathbf{1}_{\left\{\zeta_{s}<\delta\right\}}\right) \leqslant 4 C \int_{0}^{\delta} \mathrm{d} a\left(C^{\prime}+C^{\prime \prime} \int_{a^{2}}^{a} t^{-1} \mathrm{~d} t\right),
$$

which tends to 0 as $\delta \rightarrow 0$. Recalling (8) we see that the proof of Lemma 3.2 is complete.

Lemma 3.3. For every $\delta>0$,

$$
\sup _{0<\varepsilon<1} \mathbb{N}_{0}\left(\left(\varepsilon^{-4} \int_{0}^{\sigma} \mathrm{d} s \mathbf{1}_{\left\{\widehat{W}_{s}<\underline{W}+\varepsilon, \zeta_{s} \geqslant \delta\right\}}\right)^{2}\right)<\infty .
$$


Proof. We now use the case $p=2$ of Theorem 2.2 to write

$$
\mathbb{N}_{0}\left(\left(\varepsilon^{-4} \int_{0}^{\sigma} \mathrm{d} s \mathbf{1}_{\left\{\widehat{W}_{s}<\underline{W}+\varepsilon, \zeta_{s} \geqslant \delta\right\}}\right)^{2}\right)=4 \int_{\mathbb{R}_{+}^{3}} \mathrm{~d} a \mathrm{~d} b \mathrm{~d} c \mathbf{1}_{\{a+b \geqslant \delta, a+c \geqslant \delta\}} I_{\varepsilon}^{a, b, c},
$$

where

$$
\begin{aligned}
I_{\varepsilon}^{a, b, c}= & E^{a, b, c}\left[\mathbf{1}_{\{\mathcal{G} \subset] \gamma-\varepsilon, \infty[\}}\right. \\
& \left.\times \exp \left(-4\left(\int_{0}^{a} \mathrm{~d} t \mathbb{N}_{\xi_{t}}(\underline{W}>\gamma-\varepsilon)+\int_{0}^{b} \mathrm{~d} t \mathbb{N}_{\xi_{t}^{\prime}}(\underline{W}>\gamma-\varepsilon)+\int_{0}^{c} \mathrm{~d} t \mathbb{N}_{\xi_{t}^{\prime \prime}}(\underline{W}>\gamma-\varepsilon)\right)\right)\right],
\end{aligned}
$$

and, under the probability measure $P^{a, b, c}$ :

- $\left(\xi_{t}\right)_{0 \leqslant t \leqslant a}$ is a linear Brownian motion started at 0 ;

- conditionally given $\left(\xi_{t}\right)_{0 \leqslant t \leqslant a},\left(\xi_{t}^{\prime}\right)_{0 \leqslant t \leqslant b}$ and $\left(\xi_{t}^{\prime \prime}\right)_{0 \leqslant t \leqslant c}$ are independent linear Brownian motions started at $\xi_{a}$;

- $\gamma=\xi_{b}^{\prime} \vee \xi_{c}^{\prime \prime}$;

- $\mathcal{G}=\left\{\xi_{t}, 0 \leqslant t \leqslant a\right\} \cup\left\{\xi_{t}^{\prime}, 0 \leqslant t \leqslant b\right\} \cup\left\{\xi_{t}^{\prime \prime}, 0 \leqslant t \leqslant c\right\}$.

By Lemma 2.1(i),

$$
I_{\varepsilon}^{a, b, c}=E^{a, b, c}\left[\mathbf{1}_{\{\mathcal{G} \subset] \gamma-\varepsilon, \infty[\}} \exp \left(-6\left(\int_{0}^{a} \frac{\mathrm{d} t}{\left(\xi_{t}-\gamma+\varepsilon\right)^{2}}+\int_{0}^{b} \frac{\mathrm{d} t}{\left(\xi_{t}^{\prime}-\gamma+\varepsilon\right)^{2}}+\int_{0}^{c} \frac{\mathrm{d} t}{\left(\xi_{t}^{\prime \prime}-\gamma+\varepsilon\right)^{2}}\right)\right)\right] .
$$

On the event $\{\mathcal{G} \subset] \gamma-\varepsilon, \infty[\}$, we have $\left|\xi_{b}^{\prime}-\xi_{c}^{\prime \prime}\right|<\varepsilon$, and

$$
\begin{aligned}
& 0 \leqslant \xi_{t}^{\prime}-\gamma+\varepsilon \leqslant \xi_{t}^{\prime}-\xi_{b}^{\prime}+\varepsilon, \quad \forall t \in[0, b], \\
& 0 \leqslant \xi_{t}^{\prime \prime}-\gamma+\varepsilon \leqslant \xi_{t}^{\prime \prime}-\xi_{c}^{\prime \prime}+\varepsilon, \quad \forall t \in[0, c] .
\end{aligned}
$$

We use this to derive a first bound on $I_{\varepsilon}^{a, b, c}$. To write this bound in a convenient way, we introduce the following notation:

$$
\begin{array}{ll}
\eta_{t}=\xi_{a-t}-\xi_{a}, & t \in[0, a], \\
\eta_{t}^{\prime}=\xi_{b-t}^{\prime}-\xi_{b}^{\prime}, & t \in[0, b], \\
\eta_{t}^{\prime \prime}=\xi_{c-t}^{\prime \prime}-\xi_{c}^{\prime \prime}, & t \in[0, c],
\end{array}
$$

in such a way that $\eta, \eta^{\prime}, \eta^{\prime \prime}$ are three independent linear Brownian motions started at 0 under $P^{a, b, c}$, and $\gamma=-\eta_{a}-$ $\left(\eta_{b}^{\prime} \wedge \eta_{c}^{\prime \prime}\right)$. Using this notation and the preceding bounds on the event $\{\mathcal{G} \subset] \gamma-\varepsilon, \infty[\}$, we get $I_{\varepsilon}^{a, b, c} \leqslant J_{\varepsilon}^{a, b, c}$, where

$$
\begin{aligned}
J_{\varepsilon}^{a, b, c}= & E\left[\mathbf{1}\left\{\left|\eta_{b}^{\prime}-\eta_{c}^{\prime \prime}\right|<\varepsilon, \eta[0, a] \subset\right]-\left(\eta_{b}^{\prime} \wedge \eta_{c}^{\prime \prime}\right)-\varepsilon, \infty\left[, \eta^{\prime}[0, b] \subset\right]-\varepsilon, \infty\left[, \eta^{\prime \prime}[0, c] \subset\right]-\varepsilon, \infty[\}\right. \\
& \left.\times \exp \left(-6\left(\int_{0}^{a} \frac{\mathrm{d} t}{\left(\eta_{t}+\left(\eta_{b}^{\prime} \wedge \eta_{c}^{\prime \prime}\right)+\varepsilon\right)^{2}}+\int_{0}^{b} \frac{\mathrm{d} t}{\left(\eta_{t}^{\prime}+\varepsilon\right)^{2}}+\int_{0}^{c} \frac{\mathrm{d} t}{\left(\eta_{t}^{\prime \prime}+\varepsilon\right)^{2}}\right)\right)\right] .
\end{aligned}
$$

To simplify notation, we have written $E$ instead of $E^{a, b, c}$, and $\eta[0, a]$ obviously denotes the set $\left\{\eta_{t}: 0 \leqslant t \leqslant a\right\}$, with a similar notation for $\eta^{\prime}[0, b]$ and $\eta^{\prime \prime}[0, c]$.

In the preceding formula for $J_{\varepsilon}^{a, b, c}$, conditioning with respect to the pair $\left(\eta^{\prime}, \eta^{\prime \prime}\right)$ leads to a quantity depending on $y=\left(\eta_{b}^{\prime} \wedge \eta_{c}^{\prime \prime}\right)+\varepsilon$, of the form

$$
E\left[\mathbf{1}_{\{\eta[0, a] \subset]-y, \infty[\}} \exp \left(-6 \int_{0}^{a} \frac{\mathrm{d} t}{\left(\eta_{t}+y\right)^{2}}\right)\right]=E_{y}\left[\mathbf{1}_{\{\xi[0, a] \subset] 0, \infty[\}} \exp \left(-6 \int_{0}^{a} \frac{\mathrm{d} t}{\xi_{t}^{2}}\right)\right]=y^{4} E_{y}^{(9)}\left[R_{a}^{-4}\right]
$$


using Proposition 2.6 as in the proof of Lemma 3.2 above. Hence,

$$
\begin{aligned}
J_{\varepsilon}^{a, b, c}= & E\left[\mathbf{1}\left\{\left|\eta_{b}^{\prime}-\eta_{c}^{\prime \prime}\right|<\varepsilon, \eta^{\prime}[0, b] \subset\right]-\varepsilon, \infty\left[, \eta^{\prime \prime}[0, c] \subset\right]-\varepsilon, \infty[\}\right. \\
& \left.\times\left(\left(\eta_{b}^{\prime} \wedge \eta_{c}^{\prime \prime}\right)+\varepsilon\right)^{4} E_{\left(\eta_{b}^{\prime} \wedge \eta_{c}^{\prime \prime}\right)+\varepsilon}^{(9)}\left[R_{a}^{-4}\right] \exp \left(-6\left(\int_{0}^{b} \frac{\mathrm{d} t}{\left(\eta_{t}^{\prime}+\varepsilon\right)^{2}}+\int_{0}^{c} \frac{\mathrm{d} t}{\left(\eta_{t}^{\prime \prime}+\varepsilon\right)^{2}}\right)\right)\right] .
\end{aligned}
$$

Recall that our goal is to bound $\int \mathrm{d} a \mathrm{~d} b \mathrm{~d} c \mathbf{1}_{\{a+b \geqslant \delta, a+c \geqslant \delta\}} J_{\varepsilon}^{a, b, c}$. First consider the integral over the set $\{a<\delta / 2\}$. Then plainly we have $b>\delta / 2$ and $c>\delta / 2$, and we can use (4) to bound

$$
\begin{aligned}
& \int_{\{a<\delta / 2\}} \mathrm{d} a \mathrm{~d} b \mathrm{~d} c \mathbf{1}_{\{a+b \geqslant \delta, a+c \geqslant \delta\}} J_{\varepsilon}^{a, b, c} \\
& \quad \leqslant \frac{\delta}{2} \int_{] \delta / 2, \infty\left[^{2}\right.} \mathrm{d} b \mathrm{~d} c E\left[\mathbf{1}_{\left\{\eta^{\prime}[0, b] \subset\right]-\varepsilon, \infty\left[, \eta^{\prime \prime}[0, c] \subset\right]-\varepsilon, \infty[\}} \exp \left(-6\left(\int_{0}^{b} \frac{\mathrm{d} t}{\left(\eta_{t}^{\prime}+\varepsilon\right)^{2}}+\int_{0}^{c} \frac{\mathrm{d} t}{\left(\eta_{t}^{\prime \prime}+\varepsilon\right)^{2}}\right)\right)\right] \\
& \quad=\frac{\delta}{2} \int_{] \delta / 2, \infty\left[^{2}\right.} \mathrm{d} b \mathrm{~d} c\left(\varepsilon^{4} E_{\varepsilon}^{(9)}\left[R_{b}^{-4}\right]\right)\left(\varepsilon^{4} E_{\varepsilon}^{(9)}\left[R_{c}^{-4}\right]\right) \leqslant C_{\delta} \varepsilon^{8} .
\end{aligned}
$$

In the last inequality, we used the fact that, for every $y>0$,

$$
\int_{\delta / 2}^{\infty} \mathrm{d} b E_{y}^{(9)}\left[R_{b}^{-4}\right] \leqslant \int_{\delta / 2}^{\infty} \mathrm{d} b E_{0}^{(9)}\left[R_{b}^{-4}\right]=C_{\delta}^{\prime}<\infty .
$$

We still have to get a similar bound for the integral over the set $\{a \geqslant \delta / 2\}$. Applying the bound (14) with $y=$ $\left(\eta_{b}^{\prime} \wedge \eta_{c}^{\prime \prime}\right)+\varepsilon$, we see that it is enough to prove that

$$
\begin{aligned}
& \int_{\left[0, \infty\left[^{2}\right.\right.} \mathrm{d} b \mathrm{~d} c E\left[\mathbf{1}\left\{\left|\eta_{b}^{\prime}-\eta_{c}^{\prime \prime}\right|<\varepsilon, \eta^{\prime}[0, b] \subset\right]-\varepsilon, \infty\left[, \eta^{\prime \prime}[0, c] \subset\right]-\varepsilon, \infty[\}\right. \\
& \left.\quad \times\left(\left(\eta_{b}^{\prime} \wedge \eta_{c}^{\prime \prime}\right)+\varepsilon\right)^{4} \exp \left(-6\left(\int_{0}^{b} \frac{\mathrm{d} t}{\left(\eta_{t}^{\prime}+\varepsilon\right)^{2}}+\int_{0}^{c} \frac{\mathrm{d} t}{\left(\eta_{t}^{\prime \prime}+\varepsilon\right)^{2}}\right)\right)\right] \leqslant C \varepsilon^{8} .
\end{aligned}
$$

From Proposition 2.6 again, the left-hand side of (15) is equal to

$$
\varepsilon^{8} \int_{\left[0, \infty\left[^{2}\right.\right.} \mathrm{d} b \mathrm{~d} c E_{\varepsilon}^{(9)} \otimes E_{\varepsilon}^{(9)}\left[\mathbf{1}_{\left\{\left|R_{b}-\widetilde{R}_{c}\right|<\varepsilon\right\}}\left(R_{b} \wedge \widetilde{R}_{c}\right)^{4} R_{b}^{-4} \widetilde{R}_{c}^{-4}\right],
$$

where $R$ and $\widetilde{R}$ are two independent nine-dimensional Bessel processes started at $\varepsilon$ under the probability measure $P_{\varepsilon}^{(9)} \otimes P_{\varepsilon}^{(9)}$. The quantity (16) is bounded above by $\varepsilon^{8}\left(I_{1}^{\varepsilon}+I_{2}^{\varepsilon}\right)$, where

$$
\begin{aligned}
I_{1}^{\varepsilon} & =\int \mathrm{d} b \mathrm{~d} c E_{\varepsilon}^{(9)} \otimes E_{\varepsilon}^{(9)}\left[\mathbf{1}_{\left\{R_{b}<4 \varepsilon, \widetilde{R}_{c}<4 \varepsilon\right\}}\left(R_{b} \wedge \widetilde{R}_{c}\right)^{4} R_{b}^{-4} \widetilde{R}_{c}^{-4}\right] \\
& \leqslant\left(E_{\varepsilon}^{(9)}\left[\int_{0}^{\infty} \mathrm{d} b R_{b}^{-2} \mathbf{1}_{\left\{R_{b}<4 \varepsilon\right\}}\right]\right)^{2}=C<\infty
\end{aligned}
$$

and

$$
I_{2}^{\varepsilon}=\int \mathrm{d} b \mathrm{~d} c E_{\varepsilon}^{(9)} \otimes E_{\varepsilon}^{(9)}\left[\mathbf{1}_{\left\{\widetilde{R}_{c}>3 \varepsilon,\left|R_{b}-\widetilde{R}_{c}\right|<\varepsilon\right\}}\left(R_{b} \wedge \widetilde{R}_{c}\right)^{4} R_{b}^{-4} \widetilde{R}_{c}^{-4}\right]
$$


To bound $I_{2}^{\varepsilon}$, note that, if $y \geqslant 3 \varepsilon$,

$$
E_{\varepsilon}^{(9)}\left[\int_{0}^{\infty} \mathrm{d} b R_{b}^{-2} \mathbf{1}_{\left\{\left|R_{b}-y\right|<\varepsilon\right\}}\right]=C \int_{\mathbb{R}^{9}} \mathrm{~d} z\left|z-z_{\varepsilon}\right|^{-9} \mathbf{1}_{\{y-\varepsilon<|z|<y+\varepsilon\}} \leqslant C^{\prime} \frac{\varepsilon}{y},
$$

where the notation $z_{\varepsilon}$ stands for a point in $\mathbb{R}^{9}$ such that $\left|z_{\varepsilon}\right|=\varepsilon$, and we used the form of the Green function of nine-dimensional Brownian motion. It follows that

$$
I_{2}^{\varepsilon} \leqslant C^{\prime} \varepsilon E_{\varepsilon}^{(9)}\left[\int_{0}^{\infty} \mathrm{d} c R_{c}^{-3} \mathbf{1}_{\left\{R_{c} \geqslant 3 \varepsilon\right\}}\right]=C^{\prime} \varepsilon E_{0}^{(9)}\left[\int_{0}^{\infty} \mathrm{d} c R_{c}^{-3} \mathbf{1}_{\left\{R_{c} \geqslant 3 \varepsilon\right\}}\right]=C^{\prime \prime}<\infty,
$$

by a simple scaling argument. This completes the proof of the bound (15) and of Lemma 3.3.

Corollary 3.4. If $F: \Omega \rightarrow \mathbb{R}_{+}$is bounded and continuous, we have

$$
\lim _{\varepsilon \rightarrow 0} \varepsilon^{-4} \mathbb{N}_{0}\left(\left(1-\mathrm{e}^{-\sigma}\right) \int_{0}^{\sigma} \mathrm{d} s\left|F\left(W^{[s]}\right)-F\left(W^{\left[s_{*}\right]}\right)\right| \mathbf{1}_{\left\{\widehat{W}_{s}<\underline{W}+\varepsilon\right\}}\right)=0 .
$$

Proof. Thanks to Lemma 3.2, it is enough to check that, for every $\delta>0$,

$$
\lim _{\varepsilon \rightarrow 0} \varepsilon^{-4} \mathbb{N}_{0}\left(\left(1-\mathrm{e}^{-\sigma}\right) \int_{0}^{\sigma} \mathrm{d} s\left|F\left(W^{[s]}\right)-F\left(W^{\left[s_{*}\right]}\right)\right| \mathbf{1}_{\left\{\widehat{W}_{s}<\underline{W}+\varepsilon, \zeta_{s}>\delta\right\}}\right)=0 .
$$

However,

$$
\begin{aligned}
& \varepsilon^{-4} \mathbb{N}_{0}\left(\left(1-\mathrm{e}^{-\sigma}\right) \int_{0}^{\sigma} \mathrm{d} s\left|F\left(W^{[s]}\right)-F\left(W^{\left[s_{*}\right]}\right)\right| \mathbf{1}_{\left\{\widehat{W}_{s}<\underline{W}+\varepsilon, \zeta_{s}>\delta\right\}}\right) \\
& \leqslant \varepsilon^{-4} \mathbb{N}_{0}\left(\left(1-\mathrm{e}^{-\sigma}\right) \sup _{\left\{s \in[0, \sigma]: \widehat{W}_{s}<\underline{W}+\varepsilon\right\}}\left(\left|F\left(W^{[s]}\right)-F\left(W^{\left[s_{*}\right]}\right)\right|\right) \int_{0}^{\sigma} \mathrm{d} s \mathbf{1}_{\left\{\widehat{W}_{s}<\underline{W}+\varepsilon, \zeta_{s}>\delta\right\}}\right) \\
& \leqslant C_{\delta} \mathbb{N}_{0}\left(\left(1-\mathrm{e}^{-\sigma}\right)^{2} \sup _{\left\{s \in[0, \sigma]: \widehat{W}_{s}<\underline{W}+\varepsilon\right\}}\left(\left|F\left(W^{[s]}\right)-F\left(W^{\left[s_{*}\right]}\right)\right|^{2}\right)\right)^{1 / 2}
\end{aligned}
$$

by the Cauchy-Schwarz inequality and Lemma 3.3. The last quantity tends to 0 by (7) and dominated convergence.

From (5) and Corollary 3.4, the convergence of Theorem 3.1 reduces to checking that

$$
\lim _{\varepsilon \rightarrow 0} \varepsilon^{-4} \mathbb{N}_{0}\left(\varphi(\sigma) F\left(W^{\left[s_{*}\right]}\right) \int_{0}^{\sigma} \mathrm{d} s \mathbf{1}_{\left\{\widehat{W}_{s}<\underline{W}+\varepsilon\right\}}\right)=\frac{2}{21} \mathbb{N}_{0}\left(\varphi(\sigma) F\left(W^{\left[s_{*}\right]}\right)\right) .
$$

The proof of (17) will require two more lemmas. Before stating the first one, we need to introduce some notation. For $x \geqslant 0$, we suppose that we are given a Poisson point measure $\mathcal{N}=\sum_{i \in I} \delta_{\omega^{i}}$ with intensity $x \mathbb{N}_{0}$, under the probability measure $\mathbb{P}_{(x)}$. To simplify notation, we write $W_{s}^{i}=W_{s}\left(\omega^{i}\right)$. We then set

$$
\underline{\underline{W}}=\inf _{i \in I}\left(\inf _{s \geqslant 0} \widehat{W}_{s}^{i}\right)=\inf _{i \in I} \underline{W}^{i} .
$$

Lemma 3.5. For every $x>0$ and $\varepsilon>0$,

$$
\mathbb{E}_{(x)}\left[\sum_{i \in I} \int_{0}^{\sigma\left(\omega^{i}\right)} \mathrm{d} s \mathbf{1}_{\left\{\widehat{W}_{s}^{i}<\underline{\underline{W}}+\varepsilon\right\}}\right]=\varepsilon^{4} g\left(\frac{\varepsilon}{\sqrt{x}}\right),
$$

where the function $g: \mathbb{R}_{+} \rightarrow \mathbb{R}_{+}$is continuous and nonincreasing, and $g(0)=2 / 21$. 
Proof. We first recall a well-known fact about Palm distributions of Poisson point measures. If $\mathcal{M}$ is a Poisson point measure on a locally compact space, and if the intensity measure $m$ of $\mathcal{M}$ is a Radon measure, then, for every nonnegative measurable functional $\Phi$,

$$
E\left[\int \mathcal{M}(\mathrm{d} e) \Phi(e, \mathcal{M})\right]=\int m(\mathrm{~d} e) E\left[\Phi\left(e, \mathcal{M}+\delta_{e}\right)\right] .
$$

See e.g. Sections 10 and 11 in [20]. We apply this to the point measure $\mathcal{N}$ and to the function

$$
\Phi(\omega, \mathcal{N})=\int_{0}^{\sigma(\omega)} \mathrm{d} s \mathbf{1}_{\left\{\widehat{W}_{s}(\omega)<\underline{\underline{W}}+\varepsilon\right\}} .
$$

Note that $\Omega$ is not locally compact, but as a Polish space it is homeomorphic to a Borel subset of a compact metric space, so that the application of the preceding formula is easy to justify in our setting. We get

$$
\begin{aligned}
\mathbb{E}_{(x)}\left[\sum_{i \in I} \int_{0}^{\sigma\left(\omega^{i}\right)} \mathrm{d} s \mathbf{1}_{\left\{\widehat{W}_{s}^{i}<\underline{\underline{W}}+\varepsilon\right\}}\right] & =x \mathbb{N}_{0}\left(\int_{0}^{\sigma} \mathrm{d} s \mathbf{1}_{\left\{\widehat{W}_{s}<\underline{\underline{W}}+\varepsilon\right\}} \mathbb{P}_{(x)}\left[\underline{\underline{W}>a-\varepsilon]_{a=\widehat{W}_{s}}}\right)\right. \\
& =x \mathbb{N}_{0}\left(\int_{0}^{\sigma} \mathrm{d} s \mathbf{1}_{\left\{\widehat{W}_{s}<\underline{\underline{W}}+\varepsilon\right\}} \exp \left(-x \mathbb{N}_{0}(\underline{\underline{W}} \leqslant a-\varepsilon)_{a=\widehat{W}_{s}}\right)\right) \\
& =x \mathbb{N}_{0}\left(\int_{0}^{\sigma} \mathrm{d} s \mathbf{1}_{\left\{\widehat{W}_{s}<\underline{W}+\varepsilon\right\}} \exp \left(-\frac{3 x}{2\left(\widehat{W}_{s}-\varepsilon\right)^{2}}\right)\right)
\end{aligned}
$$

by Lemma 2.1(i). In a way analogous to the proof of Lemma 3.2 above, this quantity is equal to

$$
\begin{aligned}
& x \int_{0}^{\infty} \mathrm{d} a E_{0}\left[\mathbf{1}_{\{\xi[0, a] \subset] \xi_{a}-\varepsilon, \infty[\}} \exp \left(-6 \int_{0}^{a} \frac{\mathrm{d} t}{\left(\xi_{t}-\xi_{a}+\varepsilon\right)^{2}}-\frac{3 x}{2\left(\xi_{a}-\varepsilon\right)^{2}}\right)\right] \\
& \quad=x \int_{0}^{\infty} \mathrm{d} a E_{\varepsilon}\left[\mathbf{1}_{\{\xi[0, a] \subset] 0, \infty[\}} \exp \left(-6 \int_{0}^{a} \frac{\mathrm{d} t}{\xi_{t}^{2}}-\frac{3 x}{2 \xi_{a}^{2}}\right)\right] \\
& =x \varepsilon^{4} \int_{0}^{\infty} \mathrm{d} a E_{\varepsilon}^{(9)}\left[R_{a}^{-4} \exp -\frac{3 x}{2 R_{a}^{2}}\right]=\varepsilon^{4} \int_{0}^{\infty} \mathrm{d} b E_{\varepsilon / \sqrt{x}}^{(9)}\left[R_{b}^{-4} \exp -\frac{3}{2 R_{b}^{2}}\right],
\end{aligned}
$$

which gives the formula of the lemma, with

$$
g(u)=E_{u}^{(9)}\left[\int_{0}^{\infty} \mathrm{d} b R_{b}^{-4} \exp -\frac{3}{2 R_{b}^{2}}\right] .
$$

The fact that $g$ is nondecreasing follows from the strong Markov property of $R$. The continuity of $g$ is easy from a similar argument. Finally, the value of $g(0)$ is obtained from the explicit formula for the Green function of ninedimensional Brownian motion.

Recall our notation $\mathcal{E}^{] a, \infty[}$ for the $\sigma$-field generated by the Brownian snake paths before their first exit from ]a, $\infty[$, and $L^{a}$ for the total mass of the exit measure $\mathcal{Z}^{] a, \infty[}$.

Lemma 3.6. Let $a<0$. For every bounded $\mathcal{E}^{] a, \infty[}{ }_{-}$measurable function $\Phi$ on $\Omega$,

$$
\lim _{\varepsilon \rightarrow 0} \varepsilon^{-4} \mathbb{N}_{0}\left(\mathbf{1}_{\{\underline{W}<a\}} \Phi \int_{0}^{\sigma} \mathrm{d} s \mathbf{1}_{\left\{\widehat{W}_{s}<\underline{W}+\varepsilon\right\}}\right)=\frac{2}{21} \mathbb{N}_{0}\left(\mathbf{1}_{\{\underline{W}<a\}} \Phi\right) .
$$


Proof. For every $\mathrm{w} \in \mathcal{W}_{0}$, set $\tau_{a}(\mathrm{w})=\inf \{t \geqslant 0: \mathrm{w}(t) \leqslant a\}$. We first show that

$$
\lim _{\varepsilon \rightarrow 0} \varepsilon^{-4} \mathbb{N}_{0}\left(\mathbf{1}_{\{\underline{W}<a\}} \int_{0}^{\sigma} \mathrm{d} s \mathbf{1}_{\left\{\widehat{W}_{s}<\underline{W}+\varepsilon, \tau_{a}\left(W_{s}\right) \geqslant \zeta_{s}\right\}}\right)=0 .
$$

Clearly, it is enough to prove that

$$
\lim _{\varepsilon \rightarrow 0} \varepsilon^{-4} \mathbb{N}_{0}\left(\mathbf{1}_{\{\underline{W}<a\}} \int_{0}^{\sigma} \mathrm{d} s \mathbf{1}_{\left\{\widehat{W}_{s}<\underline{W}+\varepsilon, \widehat{W}_{s} \geqslant a\right\}}\right)=0 .
$$

If $\delta>0$ is fixed, we have first

$$
\begin{aligned}
\varepsilon^{-4} \mathbb{N}_{0}\left(\mathbf{1}_{\{\underline{W}<a\}} \int_{0}^{\sigma} \mathrm{d} s \mathbf{1}_{\left\{\widehat{W}_{s}<\underline{W}+\varepsilon, \widehat{W}_{s} \geqslant a\right\}} \mathbf{1}_{\left\{\zeta_{s}>\delta\right\}}\right) & \leqslant \varepsilon^{-4} \mathbb{N}_{0}\left(\mathbf{1}_{\{a-\varepsilon<\underline{W}<a\}} \int_{0}^{\sigma} \mathrm{d} s \mathbf{1}_{\left\{\widehat{W}_{s}<\underline{W}+\varepsilon, \zeta_{s}>\delta\right\}}\right) \\
& \leqslant C_{\delta} \mathbb{N}_{0}(a-\varepsilon<\underline{W}<a)^{1 / 2},
\end{aligned}
$$

by the Cauchy-Schwarz inequality and Lemma 3.3. Obviously the last quantity tends to 0 as $\varepsilon \rightarrow 0$. Then,

$$
\begin{aligned}
\varepsilon^{-4} & \mathbb{N}_{0}\left(\mathbf{1}_{\{\underline{W}<a\}} \int_{0}^{\sigma} \mathrm{d} s \mathbf{1}_{\left\{\widehat{W}_{s}<\underline{W}+\varepsilon, \widehat{W}_{s} \geqslant a\right\}} \mathbf{1}_{\left\{\zeta_{s} \leqslant \delta\right\}}\right) \\
& \leqslant \varepsilon^{-4} \mathbb{N}_{0}\left(\int_{0}^{\sigma} \mathrm{d} s \mathbf{1}_{\left\{\widehat{W}_{s}<\underline{(\underline{W}+\varepsilon) \wedge(a+\varepsilon)\}} \mathbf{1}_{\left\{\zeta_{s} \leqslant \delta\right\}}\right)}\right. \\
= & \varepsilon^{-4} \int_{0}^{\delta} \mathrm{d} t E_{0}\left[\mathbf{1}_{\left\{\xi_{t}<a+\varepsilon, \xi[0, t] \subset\right] \xi_{t}-\varepsilon, \infty[\}} \exp \left(-6 \int_{0}^{t} \frac{\mathrm{d} r}{\left(\xi_{r}-\xi_{t}+\varepsilon\right)^{2}}\right)\right] \\
= & \varepsilon^{-4} \int_{0}^{\delta} \mathrm{d} t E_{\varepsilon}\left[\mathbf{1}_{\left\{\xi_{t}>-a, \xi[0, t] \subset\right] 0, \infty[\}} \exp \left(-6 \int_{0}^{t} \frac{\mathrm{d} r}{\xi_{r}^{2}}\right)\right] \\
= & \int_{0}^{\delta} \mathrm{d} t E_{\varepsilon}^{(9)}\left[\mathbf{1}_{\left\{R_{t}>-a\right\}} R_{t}^{-4}\right] \leqslant \delta a^{-4} .
\end{aligned}
$$

The last quantity can be made arbitrarily small by choosing $\delta$ small, independently of $\varepsilon$. This completes the proof of (19) and (18).

It remains to study

$$
\begin{aligned}
\varepsilon^{-4} & \mathbb{N}_{0}\left(\mathbf{1}_{\{\underline{W}<a\}} \Phi \int_{0}^{\sigma} \mathrm{d} s \mathbf{1}_{\left\{\widehat{W}_{s}<\underline{W}+\varepsilon, \tau_{a}\left(W_{s}\right)<\zeta_{s}\right\}}\right) \\
= & \varepsilon^{-4} \mathbb{N}_{0}\left(\mathbf{1}_{\{\underline{W}<a\}} \Phi \mathbb{E}_{\left(L^{a}\right)}\left[\sum_{i \in I} \int_{0}^{\sigma\left(\omega^{i}\right)} \mathrm{d} s \mathbf{1}_{\left\{\widehat{W}_{s}^{i}<\underline{\underline{W}}+\varepsilon\right\}}\right]\right)=\mathbb{N}_{0}\left(\mathbf{1}_{\{\underline{W}<a\}} \Phi g\left(\frac{\varepsilon}{\sqrt{L^{a}}}\right)\right) .
\end{aligned}
$$

In the first equality we used the special Markov property (Theorem 2.4), and in the second one Lemma 3.5. The desired result now follows from dominated convergence.

Proof of Theorem 3.1. We already noticed that it is enough to establish (17). We first observe that

$$
\lim _{b \downarrow 0} \sup _{0<\varepsilon<1}\left(\varepsilon^{-4} \mathbb{N}_{0}\left(\mathbf{1}_{\{\underline{W} \geqslant-b\}} \varphi(\sigma) \int_{0}^{\sigma} \mathrm{d} s \mathbf{1}_{\left\{\widehat{W}_{s}<\underline{W}+\varepsilon\right\}}\right)\right)=0 .
$$


In fact, for every $\delta>0$, Lemma 3.3 gives the bound

$$
\varepsilon^{-4} \mathbb{N}_{0}\left(\mathbf{1}_{\{\underline{W} \geqslant-b\}} \varphi(\sigma) \int_{0}^{\sigma} \mathrm{d} s \mathbf{1}_{\left\{\widehat{W}_{s}<\underline{W}+\varepsilon, \zeta_{s}>\delta\right\}}\right) \leqslant C_{\delta} \mathbb{N}_{0}\left(1_{\{\underline{W} \geqslant-b\}} \varphi(\sigma)^{2}\right)^{1 / 2},
$$

and the right-hand side tends to 0 as $b \downarrow 0$ by dominated convergence. On the other hand,

$$
\sup _{0<\varepsilon<1}\left(\varepsilon^{-4} \mathbb{N}_{0}\left(\varphi(\sigma) \int_{0}^{\sigma} \mathrm{d} s \mathbf{1}_{\left\{\widehat{W}_{s}<\underline{W}+\varepsilon, \zeta_{s} \leqslant \delta\right\}}\right)\right) \underset{\delta \downarrow 0}{\longrightarrow} 0
$$

by Lemma 3.2. This completes the proof of (20).

For every $a<0$, set

$$
T_{a}=\inf \left\{s \geqslant 0: \widehat{W}_{s}=a\right\},
$$

and write $\widetilde{W}^{a}:=\widetilde{W}^{] a, \infty[}$ for the "Brownian snake truncated below level $a$ " (cf. Subsection 2.4). By definition, $\widetilde{W}^{a}$ is $\mathcal{E}^{] a, \infty[-m e a s u r a b l e . ~ F u r t h e r m o r e, ~ w e ~ h a v e ~ a l s o ~}$

$$
T_{a}=\inf \left\{s \geqslant 0: \widehat{\widetilde{W}}_{s}=a\right\}, \quad \mathbb{N}_{0} \text { a.e. }
$$

For every $s \in\left[0, \sigma\left(\widetilde{W}^{a}\right)\right]$, we can define the re-rooted snake $\widetilde{W}^{a,[s]}$ from $\widetilde{W}^{a}$, in the same way as $W^{[s]}$ was defined from $W$ in Section 2 . Note that the process $\widetilde{W}^{a,\left[T_{a}\right]}$, whose definition makes sense on the $\mathcal{E}^{] a, \infty[}$-measurable set $\left\{T_{a}<\infty\right\}$, is $\mathcal{E}^{] a, \infty[}$-measurable. Hence, Lemma 3.6 gives

$$
\lim _{\varepsilon \rightarrow 0} \varepsilon^{-4} \mathbb{N}_{0}\left(\mathbf{1}_{\{\underline{W}<a\}} \varphi\left(\sigma\left(\widetilde{W}^{a}\right)\right) F\left(\widetilde{W}^{a,\left[T_{a}\right]}\right) \int_{0}^{\sigma} \mathrm{d} s \mathbf{1}_{\left\{\widehat{W}_{s}<\underline{W}+\varepsilon\right\}}\right)=\frac{2}{21} \mathbb{N}_{0}\left(\mathbf{1}_{\{\underline{W}<a\}} \varphi\left(\sigma\left(\widetilde{W}^{a}\right)\right) F\left(\widetilde{W}^{a,\left[T_{a}\right]}\right)\right) .
$$

We fix $a<0$ of the form $a=k_{0} 2^{-n_{0}}$, where $k_{0} \in \mathbb{Z}$ and $n_{0} \in \mathbb{N}$. For every $x<0$ and $n \in \mathbb{N}$, we denote by $\{x\}_{n}$ the smallest number of the form $k 2^{-n}$, with $k \in\left[-2^{2 n}, 0\right] \cap \mathbb{Z}$, which is strictly greater than $x$. As a consequence of Lemma 3.6 (applied with $a$ replaced by $i 2^{-n}$ and with a suitable choice of $\Phi$ ), we get, for every integer $n \geqslant n_{0}$ and every $i \in \mathbb{Z}$ such that $-2^{n} \leqslant i 2^{-n}<a$,

$$
\begin{aligned}
\lim _{\varepsilon \downarrow 0} \varepsilon^{-4} \mathbb{N}_{0}\left(\mathbf { 1 } _ { \{ \underline { W } < i 2 ^ { - n } \} } \left(\varphi\left(\sigma\left(\widetilde{W}^{i 2^{-n}}\right)\right) F\left(\widetilde{W}^{i 2^{-n},\left[T_{\left.i 2^{-n}\right]}\right]}\right)\right.\right. \\
\left.\left.\quad-\varphi\left(\sigma\left(\widetilde{W}^{(i+1) 2^{-n}}\right)\right) F\left(\widetilde{W}^{(i+1) 2^{-n},\left[T_{\left.(i+1) 2^{-n}\right]}\right]}\right)\right) \int_{0}^{\sigma} \mathrm{d} s \mathbf{1}_{\left\{\widehat{W}_{s}<\underline{W}+\varepsilon\right\}}\right) \\
=\frac{2}{21} \mathbb{N}_{0}\left(\mathbf{1}_{\left\{\underline{W}<i 2^{-n}\right\}}\left(\varphi\left(\sigma\left(\widetilde{W}^{i 2^{-n}}\right)\right) F\left(\widetilde{W}^{i 2^{-n},\left[T_{\left.i 2^{-n}\right]}\right]}\right)-\varphi\left(\sigma\left(\widetilde{W}^{(i+1) 2^{-n}}\right)\right) F\left(\widetilde{W}^{(i+1) 2^{-n},\left[T_{\left.(i+1) 2^{-n}\right]}\right.}\right)\right)\right) .
\end{aligned}
$$

We sum (21) and the convergences (22) for all choices of $i \in \mathbb{Z}$ with $-2^{n} \leqslant i 2^{-n}<a$. It follows that

$$
\begin{gathered}
\lim _{\varepsilon \downarrow 0} \varepsilon^{-4} \mathbb{N}_{0}\left(\mathbf{1}_{\underline{\underline{W}<a\}}} \varphi\left(\sigma\left(\widetilde{W}^{\{\underline{W}\}_{n}}\right)\right) F\left(\widetilde{W}^{\{\underline{W}\}_{n},\left[T_{\{\underline{W}\}_{n}}\right]}\right) \int_{0}^{\sigma} \mathrm{d} s \mathbf{1}_{\left\{\widehat{W}_{s}<\underline{W}+\varepsilon\right\}}\right) \\
=\frac{2}{21} \mathbb{N}_{0}\left(\mathbf{1}_{\{\underline{W}<a\}} \varphi\left(\sigma\left(\widetilde{W}^{\{\underline{W}\}_{n}}\right)\right) F\left(\widetilde{W}^{\{\underline{W}\}_{n},\left[T_{\{\underline{W}\}_{n}}\right]}\right)\right) .
\end{gathered}
$$

Note that $\sigma\left(\widetilde{W}^{\{\underline{W}\}_{n}}\right) \leqslant \sigma$, and

$$
\sigma\left(\widetilde{W}^{\{\underline{W}\}_{n}}\right) \underset{n \rightarrow \infty}{\longrightarrow} \sigma, \quad \mathbb{N}_{0} \text { a.e. }
$$

Moreover, $T_{\{\underline{W}\}_{n}} \rightarrow T_{\underline{W}}=s_{*}$, and from the construction of re-rooted snakes it follows that

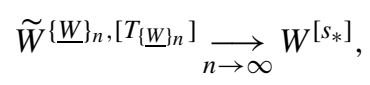


$\mathbb{N}_{0}$ a.e., in the sense of uniform convergence. By dominated convergence, we get that the right-hand side of (23) is close to

$$
\frac{2}{21} \mathbb{N}_{0}\left(\mathbf{1}_{\{\underline{W}<a\}} \varphi(\sigma) F\left(W^{\left[s_{*}\right]}\right)\right)
$$

when $n \rightarrow \infty$.

Using (20) and (23), we see that the proof of (17) will be complete if we can verify that

$$
\sup _{0<\varepsilon<1}\left(\varepsilon^{-4} \mathbb{N}_{0}\left(\mathbf{1}_{\{\underline{W}<a\}}\left|\varphi\left(\sigma\left(\widetilde{W}^{\{\underline{W}\}_{n}}\right)\right) F\left(\widetilde{W}^{\{\underline{W}\}_{n},\left[T_{\left.\{\underline{W}\}_{n}\right]}\right]}\right)-\varphi(\sigma) F\left(W^{\left[s_{*}\right]}\right)\right| \int_{0}^{\sigma} \mathrm{d} s \mathbf{1}_{\left\{\widehat{W}_{s}<\underline{W}+\varepsilon\right\}}\right)\right)
$$

tends to 0 as $n \rightarrow \infty$. This is easy by decomposing the set $\left\{\widehat{W}_{s}<\underline{W}+\varepsilon\right\}$ as

$$
\left\{\widehat{W}_{s}<\underline{W}+\varepsilon, \zeta_{s} \leqslant \delta\right\} \cup\left\{\widehat{W}_{s}<\underline{W}+\varepsilon, \zeta_{s}>\delta\right\}
$$

and using Lemma 3.2 for the first term and Lemma 3.3, together with the Cauchy-Schwarz inequality, for the second one, as we did previously. This completes the proof of (17) and of Theorem 3.1.

Proof of Theorems 1.1 and 1.2. Both Theorems 1.1 and 1.2 follow from the convergence

$$
\lim _{\varepsilon \rightarrow 0} \varepsilon^{-4} \mathbb{N}_{0}^{(1)}\left(F(W) \mathbf{1}_{\{\underline{W}>-\varepsilon\}}\right)=\frac{2}{21} \mathbb{N}_{0}^{(1)}\left(F\left(W^{\left[s_{*}\right]}\right)\right),
$$

which holds for every bounded continuous function $F$ on $\Omega=C\left(\mathbb{R}_{+}, \mathcal{W}\right.$ ) (take $F=1$ to recover the first assertion of Theorem 1.1). We will now derive (24) from Theorem 3.1.

For every $\lambda>0$, let us introduce the scaling operator $\theta_{\lambda}$ defined on $\Omega$ by

$$
\begin{aligned}
& \zeta_{s} \circ \theta_{\lambda}=\lambda^{1 / 2} \zeta_{s / \lambda}, \\
& W_{s} \circ \theta_{\lambda}(t)=\lambda^{1 / 4} W_{s / \lambda}\left(\lambda^{-1 / 2} t\right) .
\end{aligned}
$$

Note that, for every $r>0$, the image of $\mathbb{N}_{0}^{(r)}$ under $\theta_{1 / r}$ is $\mathbb{N}_{0}^{(1)}$.

Let $\delta \in] 0,1[$. It follows from Theorem 3.1 that the law of the pair $(\sigma, W)$ under

$$
\mu_{\varepsilon, \delta}:=\varepsilon^{-4} \mathbb{N}_{0}(\cdot \cap\{\underline{W}>-\varepsilon, 1-\delta<\sigma<1\})
$$

converges weakly as $\varepsilon \rightarrow 0$ towards the law of $\left(\sigma, W^{\left[s_{*}\right]}\right)$ under the measure $\mu_{\delta}$ having density $2 /(21 \sigma)$ with respect to $\mathbb{N}_{0}(\cdot \cap\{1-\delta<\sigma<1\})$.

Since the mapping $(r, \omega) \rightarrow \theta_{r} \omega$ is continuous, it follows that the law of $W \circ \theta_{1 / \sigma}$ under $\mu_{\varepsilon, \delta}$ converges as $\varepsilon \rightarrow 0$ towards the law of $W^{\left[s_{*}\right]} \circ \theta_{1 / \sigma}$ under $\mu_{\delta}$. Thus,

$$
\lim _{\varepsilon \rightarrow 0} \mu_{\varepsilon, \delta}\left(F\left(W \circ \theta_{1 / \sigma}\right)\right)=\mu_{\delta}\left(F\left(W^{\left[s_{*}\right]} \circ \theta_{1 / \sigma}\right)\right),
$$

or equivalently

$$
\lim _{\varepsilon \rightarrow 0} \varepsilon^{-4} \mathbb{N}_{0}\left(\mathbf{1}_{\{\underline{W}>-\varepsilon, 1-\delta<\sigma<1\}} F\left(W \circ \theta_{1 / \sigma}\right)\right)=\mathbb{N}_{0}\left(\frac{2}{21 \sigma} \mathbf{1}_{\{1-\delta<\sigma<1\}} F\left(W^{\left[s_{*}\right]} \circ \theta_{1 / \sigma}\right)\right) .
$$

Since the density of $\sigma$ under $\mathbb{N}_{0}$ is $(8 \pi)^{-1 / 2} s^{-3 / 2}$, this can be rewritten as

$$
\lim _{\varepsilon \rightarrow 0} \varepsilon^{-4} \int_{1-\delta}^{1} \frac{\mathrm{d} r}{2 \sqrt{2 \pi r^{3}}} \mathbb{N}_{0}^{(r)}\left(F\left(W \circ \theta_{1 / r}\right) \mathbf{1}_{\{\underline{W}>-\varepsilon\}}\right)=\frac{2}{21} \int_{1-\delta}^{1} \frac{\mathrm{d} r}{2 \sqrt{2 \pi r^{5}}} \mathbb{N}_{0}^{(r)}\left(F\left(W^{\left[s_{*}\right]} \circ \theta_{1 / r}\right)\right) .
$$

Now observe that $\underline{W}=r^{1 / 4} \underline{W} \circ \theta_{1 / r}$, and recall that the image of $\mathbb{N}_{0}^{(r)}$ under $\theta_{1 / r}$ is $\mathbb{N}_{0}^{(1)}$ to get

$$
\lim _{\varepsilon \rightarrow 0} \varepsilon^{-4} \int_{1-\delta}^{1} \frac{\mathrm{d} r}{2 \sqrt{2 \pi r^{3}}} \mathbb{N}_{0}^{(1)}\left(F(W) \mathbf{1}_{\left\{\underline{W}>-r^{-1 / 4} \varepsilon\right\}}\right)=\frac{2}{21}\left(\int_{1-\delta}^{1} \frac{\mathrm{d} r}{2 \sqrt{2 \pi r^{5}}}\right) \mathbb{N}_{0}^{(1)}\left(F\left(W^{\left[s_{*}\right]}\right)\right) .
$$


Without loss of generality we can assume that $F \geqslant 0$. Since $r^{-1 / 4} \varepsilon \geqslant \varepsilon$ for $1-\delta \leqslant r \leqslant 1$, taking $\delta$ small in the preceding convergence leads to

$$
\limsup _{\varepsilon \rightarrow 0} \varepsilon^{-4} \mathbb{N}_{0}^{(1)}\left(F(W) \mathbf{1}_{\{\underline{W}>-\varepsilon\}}\right) \leqslant \frac{2}{21} \mathbb{N}_{0}^{(1)}\left(F\left(W^{\left[s_{*}\right]}\right)\right) .
$$

By arguing with the constraint $1<\sigma<1+\delta$ instead of $1-\delta<\sigma<1$, we get the analogous lower bound for the liminf behavior. This completes the proof of (24).

We conclude this section with another approximation of the conditioned measure $\overline{\mathbb{N}}_{0}^{(1)}$, which is similar to Theorem 1.1 but much easier to obtain. Recall that the random measure $\mathcal{Z}$ on $\mathbb{R}$ defined under $\mathbb{N}_{0}^{(1)}$ by the formula

$$
\langle\mathcal{Z}, \varphi\rangle=\int_{0}^{1} \mathrm{~d} s \varphi\left(\widehat{W}_{s}\right)
$$

is one-dimensional ISE.

Proposition 3.7. For any bounded continuous function $F$ on $\Omega$,

$$
\left.\left.\lim _{\varepsilon \rightarrow 0} \mathbb{N}_{0}^{(1)}(F(W) \mid \mathcal{Z}(]-\infty, 0]\right) \leqslant \varepsilon\right)=\overline{\mathbb{N}}_{0}^{(1)}(F) .
$$

Proof. As a simple consequence of the re-rooting invariance, the law of $\mathcal{Z}(]-\infty, 0])$ under $\mathbb{N}_{0}^{(1)}$ is uniform over $[0,1]$ (this fact was already observed in Section 3.2 of [6]). Hence,

$$
\begin{aligned}
\left.\left.\mathbb{N}_{0}^{(1)}(F(W) \mid \mathcal{Z}(]-\infty, 0]\right) \leqslant \varepsilon\right) & =\varepsilon^{-1} \mathbb{N}_{0}^{(1)}\left(F(W) \mathbf{1}_{\{\mathcal{Z}(]-\infty, 0]) \leqslant \varepsilon\}}\right) \\
& =\varepsilon^{-1} \mathbb{N}_{0}^{(1)}\left(\int_{0}^{1} \mathrm{~d} s F\left(W^{[s]}\right) \mathbf{1}_{\left.\left.\left\{\mathcal{Z}(]-\infty, \widehat{W}_{s}\right]\right) \leqslant \varepsilon\right\}}\right),
\end{aligned}
$$

where the second equality follows from the re-rooting theorem (Theorem 2.3) and the remark after this statement. Now, since the measure $\mathcal{Z}$ has no atoms,

$$
\int_{0}^{1} \mathrm{~d} s \mathbf{1}_{\left.\left.\left\{\mathcal{Z}(]-\infty, \widehat{W}_{s}\right]\right) \leqslant \varepsilon\right\}}=\int \mathcal{Z}(\mathrm{d} y) \mathbf{1}_{\{\mathcal{Z}(]-\infty, y]) \leqslant \varepsilon\}}=\varepsilon
$$

and on the other hand the closed sets $\left.\left.\left\{s \in[0,1]: \mathcal{Z}(]-\infty, \widehat{W}_{s}\right]\right) \leqslant \varepsilon\right\}$ decrease to the singleton $\left\{s_{*}\right\}$ as $\varepsilon \downarrow 0, \mathbb{N}_{0}^{(1)}$ a.e. It follows that

$$
\lim _{\varepsilon \rightarrow 0} \varepsilon^{-1} \int_{0}^{1} \mathrm{~d} s F\left(W^{[s]}\right) \mathbf{1}_{\left.\left.\left\{\mathcal{Z}(]-\infty, \widehat{W}_{s}\right]\right) \leqslant \varepsilon\right\}}=F\left(W^{\left[s_{*}\right]}\right)
$$

$\mathbb{N}_{0}^{(1)}$ a.e. Using dominated convergence and Theorem 1.2, we get Proposition 3.7.

Remark. Up to some point, the approximation given in Proposition 3.7 may seem as "reasonable" as the one we used in Theorem 1.1 to define $\overline{\mathbb{N}}_{0}^{(1)}$. In applications such that the ones developed in [25], the approximation given by Theorem 1.1 turns out to be more useful.

\section{Other conditionings}

Motivated by Theorem 3.1, we define a $\sigma$-finite measure $\overline{\mathbb{N}}_{0}$ on $\Omega$ by setting

$$
\overline{\mathbb{N}}_{0}(F)=\mathbb{N}_{0}\left(\frac{1}{\sigma} F\left(W^{\left[s_{*}\right]}\right)\right) .
$$


Theorem 3.1 shows that, up to the multiplicative constant $2 / 21, \overline{\mathbb{N}}_{0}$ is the limit in an appropriate sense of the measures $\varepsilon^{-4} \mathbb{N}_{0}(\cdot \cap\{\underline{W}>-\varepsilon\})$ as $\varepsilon \rightarrow 0$. We have also

$$
\overline{\mathbb{N}}_{0}(F)=\int_{0}^{\infty} \frac{\mathrm{d} r}{2 \sqrt{2 \pi r^{5}}} \mathbb{N}_{0}^{(r)}\left(F\left(W^{\left[s_{*}\right]}\right)\right)=\int_{0}^{\infty} \frac{\mathrm{d} r}{2 \sqrt{2 \pi r^{5}}} \overline{\mathbb{N}}_{0}^{(r)}(F),
$$

where $\overline{\mathbb{N}}_{0}^{(r)}$ can be defined equivalently as the law of $W^{\left[s_{*}\right]}$ under $\mathbb{N}_{0}^{(r)}$, or as the image of $\overline{\mathbb{N}}_{0}^{(1)}$ under the scaling operator $\theta_{r}$.

We will now describe a different approach to $\overline{\mathbb{N}}_{0}$, which involves conditioning the Brownian snake excursion on its height $H=\sup _{s \geqslant 0} \zeta_{s}$, rather than on its length as in Theorem 1.1. This will give more insight in the behavior of the Brownian snake under $\overline{\mathbb{N}}_{0}$. Eventually, this will lead to a construction of a Brownian snake excursion with infinite length conditioned to stay on the positive side. We rely on some ideas from [1].

For every $h>0$, we set $\mathbb{N}_{0}^{h}=\mathbb{N}_{0}(\cdot \mid H=h)$. Then,

$$
\mathbb{N}_{0}=\int_{0}^{\infty} \frac{\mathrm{d} h}{2 h^{2}} \mathbb{N}_{0}^{h}
$$

From Theorem 1 in [1] we know that there exists a constant $c_{0}>0$ such that

$$
\lim _{\varepsilon \rightarrow 0} \varepsilon^{-4} \mathbb{N}_{0}^{1}(\underline{W}>-\varepsilon)=c_{0} .
$$

A simple scaling argument then implies that, for every $h>0$,

$$
\lim _{\varepsilon \rightarrow 0} \varepsilon^{-4} \mathbb{N}_{0}^{h}(\underline{W}>-\varepsilon)=\frac{c_{0}}{h^{2}} .
$$

Theorem 4.1. For every $h>0$, there exists a probability measure $\overline{\mathbb{N}}_{0}^{h}$ on $\Omega$ such that

$$
\lim _{\varepsilon \rightarrow 0} \mathbb{N}_{0}^{h}(\cdot \mid \underline{W}>-\varepsilon)=\overline{\mathbb{N}}_{0}^{h}
$$

in the sense of weak convergence on the space of probability measures on $\Omega$. Moreover,

$$
\overline{\mathbb{N}}_{0}=\frac{21 c_{0}}{4} \int_{0}^{\infty} \frac{\mathrm{d} h}{h^{4}} \overline{\mathbb{N}}_{0}^{h}
$$

Remark. Our proof of the first part of Theorem 4.1 does not use Section 3. This proof thus gives another approach to the conditioned measure $\overline{\mathbb{N}}_{0}$, which does not depend on the re-rooting method that played a crucial role in Section 3 .

Before proving Theorem 4.1, we will establish an important preliminary result. We first introduce some notation. Following [1], we set for every $\varepsilon>0$,

$$
f(\varepsilon)=\mathbb{N}_{0}^{1}(\underline{W}>-\varepsilon)
$$

and, for every $x>0$,

$$
G(x)=4 \int_{0}^{x} u(1-f(u)) \mathrm{d} u .
$$

The function $G$ is obviously nondecreasing. It is also bounded since

$$
G(\infty)=4 \int_{0}^{\infty} u \mathbb{N}_{0}^{1}(\underline{W} \leqslant-u) \mathrm{d} u=2 \int_{0}^{\infty} r^{-2} \mathbb{N}_{0}^{r}(\underline{W} \leqslant-1) \mathrm{d} r=4 \mathbb{N}_{0}(\underline{W} \leqslant-1)=6
$$

by a scaling argument and Lemma 2.1(i).

By well-known properties of Brownian excursions, there exists $\mathbb{N}_{0}^{h}$ a.s. a unique time $\left.\alpha \in\right] 0, \sigma$ [ such that $\zeta_{\alpha}=h$. The next proposition discusses the law of $W_{\alpha}$ under $\mathbb{N}_{0}^{h}(\cdot \mid \underline{W}>-\varepsilon)$. 
Proposition 4.2. Let $\Phi$ be a bounded continuous function on $\mathcal{W}$. Then,

$$
\lim _{\varepsilon \downarrow 0} \varepsilon^{-4} \mathbb{N}_{0}^{h}\left(\Phi\left(W_{\alpha}\right) \mathbf{1}_{\{\underline{W}>-\varepsilon\}}\right)=E_{0}^{(9)}\left[\Phi\left(R_{t}, 0 \leqslant t \leqslant h\right) R_{h}^{-4} \exp \left(\int_{0}^{h} \frac{\mathrm{d} t}{R_{t}^{2}} G\left(\frac{R_{t}}{\sqrt{h-t}}\right)\right)\right] .
$$

Remarks. (i) From the bound $G(x) \leqslant 6 \wedge\left(2 x^{2}\right)$, it is immediate to verify that

$$
\int_{0}^{h} \frac{\mathrm{d} t}{R_{t}^{2}} G\left(\frac{R_{t}}{\sqrt{h-t}}\right)<\infty, \quad P_{0}^{(9)} \text { a.s. }
$$

(ii) By taking $\Phi=1$, we see that the constant $c_{0}$ in (25) is given by

$$
c_{0}=E_{0}^{(9)}\left[R_{1}^{-4} \exp \left(\int_{0}^{1} \frac{\mathrm{d} t}{R_{t}^{2}} G\left(\frac{R_{t}}{\sqrt{1-t}}\right)\right)\right]
$$

as it was already observed in [1]. The fact that the quantity in the right-hand side is finite follows from the proof below.

Proof. Our main tool is Williams' decomposition of the Brownian excursion at its maximum (see e.g. Theorem XII.4.5 in [28]). For every $s \geqslant 0$, we set

$$
\rho_{s}=\zeta_{s \wedge \alpha}, \quad \rho_{s}^{\prime}=\zeta_{(\sigma-s) \vee \alpha} .
$$

Under the probability measure $\mathbb{N}_{0}^{h}$, the processes $\left(\rho_{s}\right)_{s \geqslant 0}$ and $\left(\rho_{s}^{\prime}\right)_{s} \geqslant 0$ are two independent three-dimensional Bessel processes started at 0 and stopped at their first hitting time of $h$.

We also need to introduce the excursions of $\rho$ and $\rho^{\prime}$ above their future infimum. Set

$$
\underline{\rho} s=\inf _{r \geqslant s} \rho_{r}
$$

and let $\left(a_{j}, b_{j}\right), j \in J$, be the connected components of the open set $\left\{s \geqslant 0: \rho_{s}>\underline{\rho}_{s}\right\}$. For every $j \in J$, define

$$
\begin{aligned}
& \zeta_{s}^{j}=\rho_{\left(a_{j}+s\right) \wedge b_{j}}-\rho_{a_{j}}, \quad s \geqslant 0, \\
& h_{j}=\rho_{a_{j}} .
\end{aligned}
$$

Then, by excursion theory,

$$
\sum_{j \in J} \delta_{\left(h_{j}, \zeta^{j}\right)}(\mathrm{d} r \mathrm{~d} e)
$$

is a Poisson point measure on $\mathbb{R}_{+} \times C\left(\mathbb{R}_{+}, \mathbb{R}_{+}\right)$with intensity

$$
2 \mathbf{1}_{[0, h]}(r) \mathbf{1}_{[0, h-r]}(H(e)) \mathrm{d} r n(\mathrm{~d} e)
$$

where $H(e)=\sup _{s \geqslant 0} e(s)$ as previously. The same result obviously holds for the analogous point measure

$$
\sum_{j \in J^{\prime}} \delta_{\left(h_{j}^{\prime}, \zeta^{\prime j}\right)}(\mathrm{d} r \mathrm{~d} e)
$$

obtained by replacing $\rho$ with $\rho^{\prime}$.

We can combine the preceding assertions with the spatial displacements of the Brownian snake, in a way very similar to the proof of Lemma V.5 in [24]. For every $j \in J$, we set

$$
W_{s}^{j}(t)=W_{\left(a_{j}+s\right) \wedge b_{j}}\left(h_{j}+t\right)-\widehat{W}_{a_{j}}, \quad 0 \leqslant t \leqslant \zeta_{s}^{j}, s \geqslant 0 .
$$

Note that by the properties of the Brownian snake $\widehat{W}_{a_{j}}=\widehat{W}_{b_{j}}=W_{\alpha}\left(h_{j}\right)$. Then,

$$
\mathcal{N}:=\sum_{j \in J} \delta_{\left(h_{j}, W^{j}\right)}
$$


is under $\mathbb{N}_{0}^{h}$ a Poisson point measure on $\mathbb{R}_{+} \times \Omega$, with intensity

$$
2 \mathbf{1}_{[0, h]}(r) \mathbf{1}_{[0, h-r]}(H(\omega)) \mathrm{d} r \mathbb{N}_{0}(\mathrm{~d} \omega) .
$$

The same holds for the analogous point measure

$$
\mathcal{N}^{\prime}:=\sum_{j \in J^{\prime}} \delta_{\left(h_{j}^{\prime}, W^{\prime j}\right)} .
$$

Moreover $\mathcal{N}$ and $\mathcal{N}^{\prime}$ are independent and the pair $\left(\mathcal{N}, \mathcal{N}^{\prime}\right)$ is independent of $W_{\alpha}$. All these assertions easily follow from properties of the Brownian snake.

Now note that the range of the Brownian snake under $\mathbb{N}_{0}^{h}$ can be decomposed as

$$
\left\{W_{\alpha}(t): 0 \leqslant t \leqslant h\right\} \cup\left(\bigcup_{j \in J}\left(W_{\alpha}\left(h_{j}\right)+\mathcal{R}\left(W^{j}\right)\right)\right) \cup\left(\bigcup_{j \in J^{\prime}}\left(W_{\alpha}\left(h_{j}^{\prime}\right)+\mathcal{R}\left(W^{\prime j}\right)\right)\right) .
$$

Using this observation and conditioning with respect to $W_{\alpha}$, we get

$$
\begin{aligned}
\mathbb{N}_{0}^{h}\left(\Phi\left(W_{\alpha}\right) \mathbf{1}_{\{\underline{W}>-\varepsilon\}}\right) & =\mathbb{N}_{0}^{h}\left(\Phi\left(W_{\alpha}\right) \mathbf{1}_{\left\{W_{\alpha}(t)>-\varepsilon, 0 \leqslant t \leqslant h\right\}} \exp \left(-4 \int_{0}^{h} \mathrm{~d} t \mathbb{N}_{W_{\alpha}(t)}(H<h-t, \underline{W} \leqslant-\varepsilon)\right)\right) \\
& =E_{0}\left[\Phi\left(\xi_{t}, 0 \leqslant t \leqslant h\right) \mathbf{1}_{\{\xi[0, h] \subset]-\varepsilon, \infty[\}} \exp \left(-4 \int_{0}^{h} \mathrm{~d} t \mathbb{N}_{\xi_{t}}(H<h-t, \underline{W} \leqslant-\varepsilon)\right)\right] .
\end{aligned}
$$

Then, for every $x>-\varepsilon$,

$$
\mathbb{N}_{x}(H<h-t, \underline{W} \leqslant-\varepsilon)=\int_{0}^{h-t} \frac{\mathrm{d} u}{2 u^{2}} \mathbb{N}_{x}^{u}(\underline{W} \leqslant-\varepsilon)=\int_{0}^{h-t} \frac{\mathrm{d} u}{2 u^{2}}\left(1-f\left(\frac{x+\varepsilon}{\sqrt{u}}\right)\right)
$$

and we obtain

$$
\begin{aligned}
& \mathbb{N}_{0}^{h}\left(\Phi\left(W_{\alpha}\right) \mathbf{1}_{\{\underline{W}>-\varepsilon\}}\right) \\
& =E_{0}\left[\Phi\left(\xi_{t}, 0 \leqslant t \leqslant h\right) \mathbf{1}_{\{\xi[0, h] \subset]-\varepsilon, \infty[\}} \exp \left(-2 \int_{0}^{h} \mathrm{~d} t \int_{0}^{h-t} \frac{\mathrm{d} u}{u^{2}}\left(1-f\left(\frac{\xi_{t}+\varepsilon}{\sqrt{u}}\right)\right)\right)\right] \\
& =E_{\varepsilon}\left[\Phi\left(\xi_{t}-\varepsilon, 0 \leqslant t \leqslant h\right) \mathbf{1}_{\{\xi[0, h] \subset] 0, \infty[\}} \exp \left(-2 \int_{0}^{h} \mathrm{~d} t \int_{0}^{h-t} \frac{\mathrm{d} u}{u^{2}}\left(1-f\left(\frac{\xi_{t}}{\sqrt{u}}\right)\right)\right)\right] .
\end{aligned}
$$

For every $x>0$, the change of variable $v=x / \sqrt{u}$ gives

$$
\int_{0}^{h-t} \frac{\mathrm{d} u}{u^{2}}\left(1-f\left(\frac{x}{\sqrt{u}}\right)\right)=2 x^{-2} \int_{x / \sqrt{h-t}}^{\infty} \mathrm{d} v v(1-f(v))=x^{-2}\left(3-\frac{1}{2} G\left(\frac{x}{\sqrt{h-t}}\right)\right) .
$$

By substituting this into (28) and using Proposition 2.6 once more, we get

$$
\begin{aligned}
\mathbb{N}_{0}^{h}\left(\Phi\left(W_{\alpha}\right) \mathbf{1}_{\{\underline{W}>-\varepsilon\}}\right) & =E_{\varepsilon}\left[\Phi\left(\xi_{t}-\varepsilon, 0 \leqslant t \leqslant h\right) \mathbf{1}_{\{\xi[0, h] \subset] 0, \infty[\}} \exp \left(-6 \int_{0}^{h} \frac{\mathrm{d} t}{\xi_{t}^{2}}+\int_{0}^{h} \frac{\mathrm{d} t}{\xi_{t}^{2}} G\left(\frac{\xi_{t}}{\sqrt{h-t}}\right)\right)\right] \\
& =\varepsilon^{4} E_{\varepsilon}^{(9)}\left[\Phi\left(R_{t}-\varepsilon, 0 \leqslant t \leqslant h\right) R_{h}^{-4} \exp \left(\int_{0}^{h} \frac{\mathrm{d} t}{R_{t}^{2}} G\left(\frac{R_{t}}{\sqrt{h-t}}\right)\right)\right] .
\end{aligned}
$$


In view of (29), the proof of Proposition 4.2 reduces to checking that

$$
\begin{gathered}
\lim _{\varepsilon \downarrow 0} E_{\varepsilon}^{(9)}\left[\Phi\left(R_{t}-\varepsilon, 0 \leqslant t \leqslant h\right) R_{h}^{-4} \exp \left(\int_{0}^{h} \frac{\mathrm{d} t}{R_{t}^{2}} G\left(\frac{R_{t}}{\sqrt{h-t}}\right)\right)\right] \\
=E_{0}^{(9)}\left[\Phi\left(R_{t}, 0 \leqslant t \leqslant h\right) R_{h}^{-4} \exp \left(\int_{0}^{h} \frac{\mathrm{d} t}{R_{t}^{2}} G\left(\frac{R_{t}}{\sqrt{h-t}}\right)\right)\right] .
\end{gathered}
$$

This follows from a dominated convergence argument, which at the same time will prove that the quantity in the right-hand side of (30) is well-defined. Note that we may define on a common probability space, a nine-dimensional Bessel process $X^{\varepsilon}=\left(X_{t}^{\varepsilon}, t \geqslant 0\right)$ started at $\varepsilon$, for every $\varepsilon \geqslant 0$, in such a way that the inequality $X^{\varepsilon} \geqslant X^{0}$ holds a.s. for every $\varepsilon>0$. Since

$$
G\left(\frac{X_{t}^{\varepsilon}}{\sqrt{h-t}}\right) \leqslant \frac{4\left(X_{t}^{\varepsilon}\right)^{2}}{h}, \quad \forall t \in[0, h / 2]
$$

we first get

$$
\begin{aligned}
\left(X_{h}^{\varepsilon}\right)^{-4} \exp \left(\int_{0}^{h} \frac{\mathrm{d} t}{\left(X_{t}^{\varepsilon}\right)^{2}} G\left(\frac{X_{t}^{\varepsilon}}{\sqrt{h-t}}\right)\right) & \leqslant \mathrm{e}^{2}\left(X_{h}^{\varepsilon}\right)^{-4} \exp \left(\int_{h / 2}^{h} \frac{\mathrm{d} t}{\left(X_{t}^{\varepsilon}\right)^{2}} G\left(\frac{X_{t}^{\varepsilon}}{\sqrt{h-t}}\right)\right) \\
& \leqslant \mathrm{e}^{2}\left(X_{h}^{0}\right)^{-4} \exp \left(6 \int_{h / 2}^{h} \frac{\mathrm{d} t}{\left(X_{t}^{0}\right)^{2}}\right),
\end{aligned}
$$

using the bounds $G \leqslant 6$ and $X^{\varepsilon} \geqslant X^{0}$. Then, an application of Itô's formula shows that

$$
\left(X_{t}^{0}\right)^{-4} \exp \left(6 \int_{h / 2}^{t} \frac{\mathrm{d} r}{\left(X_{r}^{0}\right)^{2}}\right)
$$

is a local martingale on the time interval $[h / 2, \infty[$, and so

$$
E\left[\left(X_{h}^{0}\right)^{-4} \exp \left(6 \int_{h / 2}^{h} \frac{\mathrm{d} t}{\left(X_{t}^{0}\right)^{2}}\right)\right] \leqslant E\left[\left(X_{h / 2}^{0}\right)^{-4}\right]<\infty .
$$

Together with (31), this shows that the random variables appearing in the left-hand side of (30) are uniformly integrable. The convergence (30) easily follows.

Proof of Theorem 4.1. We first explain how the first part of Theorem 4.1 can be deduced from Proposition 4.2. Recall the notation $\left(\mathcal{N}, \mathcal{N}^{\prime}\right)$ from the proof of this proposition. We first observe that we can find a measurable functional $\Gamma$ such that

$$
W=\Gamma\left(W_{\alpha}, \mathcal{N}, \mathcal{N}^{\prime}\right), \quad \mathbb{N}_{0}^{h} \text { a.s. }
$$

Let us make this functional more explicit. We have first

$$
\alpha=\sum_{j \in J} \sigma\left(W^{j}\right) .
$$

For every $\ell \in[0, h]$, we set

$$
\tau_{\ell}=\sum_{j \in J} \mathbf{1}_{\left\{h_{j} \leqslant \ell\right\}} \sigma\left(W^{j}\right) .
$$

Then, if $s \in[0, \alpha]$, there is a unique $\ell$ such that $\tau_{\ell-} \leqslant s \leqslant \tau_{\ell}$, and: 
- Either there is a (unique) $j \in J$ such that $\ell=h_{j}$, and

$$
\begin{aligned}
& \zeta_{s}=\ell+\zeta_{s-\tau_{\ell-},}^{j} \\
& W_{s}(t)= \begin{cases}W_{\alpha}(t) & \text { if } t \leqslant \ell, \\
W_{\alpha}(\ell)+W_{s-\tau_{\ell-}}^{j}(t-\ell) & \text { if } \ell<t \leqslant \zeta_{s} ;\end{cases}
\end{aligned}
$$

- Or there is no such $j$, and

$$
\begin{aligned}
& \zeta_{s}=\ell, \\
& W_{s}(t)=W_{\alpha}(t), \quad t \leqslant \ell .
\end{aligned}
$$

The previous formulas identify $\left(W_{s}, 0 \leqslant s \leqslant \alpha\right)$ as a measurable function of the pair $\left(W_{\alpha}, \mathcal{N}\right)$, and in a similar way we can recover $\left(W_{\sigma-s}, 0 \leqslant s \leqslant \sigma-\alpha\right)$ as the same measurable function of $\left(W_{\alpha}, \mathcal{N}^{\prime}\right)$.

To simplify notation, write $\mathbb{N}^{h,(\varepsilon)}$ for the conditional probability $\mathbb{N}_{0}^{h}(\cdot \mid \underline{W}>-\varepsilon)$. From elementary properties of Poisson measures, we get that under the probability measure $\mathbb{N}_{0}^{h,(\varepsilon)}$ and conditionally given $W_{\alpha}$, the point measures $\mathcal{N}$ and $\mathcal{N}^{\prime}$ are independent and Poisson with intensity

$$
\mu_{\varepsilon}^{h}\left(W_{\alpha} ; \mathrm{d} r \mathrm{~d} \omega\right):=2 \mathbf{1}_{[0, h]}(r) \mathbf{1}_{[0, h-r]}(H(\omega)) \mathbf{1}_{\{\mathcal{R}(\omega) \subset]-\varepsilon-W_{\alpha}(r), \infty[\}} \mathrm{d} r \mathbb{N}_{0}(\mathrm{~d} \omega) .
$$

As a consequence of Proposition 4.2, the law of $W_{\alpha}$ under $\mathbb{N}_{0}^{h,(\varepsilon)}$ converges as $\varepsilon \rightarrow 0$ to the law of the process $Y^{h}=\left(Y_{t}^{h}, 0 \leqslant t \leqslant h\right)$ such that

$$
E\left[\Phi\left(Y^{h}\right)\right]=\frac{h^{2}}{c_{0}} E_{0}^{(9)}\left[\Phi\left(R_{t}, 0 \leqslant t \leqslant h\right) R_{h}^{-4} \exp \left(\int_{0}^{h} \frac{\mathrm{d} t}{R_{t}^{2}} G\left(\frac{R_{t}}{\sqrt{h-t}}\right)\right)\right] .
$$

Suppose that on the same probability space where $Y^{h}$ is defined, we are also given two random point measures $\mathcal{M}$ and $\mathcal{M}^{\prime}$ on $\mathbb{R}_{+} \times \Omega$, which conditionally given $Y^{h}$ are independent Poisson point measures with intensity

$$
\mu_{0}^{h}\left(Y^{h} ; \mathrm{d} r \mathrm{~d} \omega\right):=2 \mathbf{1}_{[0, h]}(r) \mathbf{1}_{[0, h-r]}(H(\omega)) \mathbf{1}_{\{\mathcal{R}(\omega) \subset]-Y_{r}^{h}, \infty[\}} \mathrm{d} r \mathbb{N}_{0}(\mathrm{~d} \omega) .
$$

From the continuity properties of the "reconstruction mapping" $\Gamma$, it should now be clear that the probability measures $\mathbb{N}^{h,(\varepsilon)}$ converge as $\varepsilon \rightarrow 0$ to the measure $\overline{\mathbb{N}}_{0}^{h}$ defined as the law of $\Gamma\left(Y^{h}, \mathcal{M}, \mathcal{M}^{\prime}\right)$. Here we leave some easy technical details to the reader.

Let us prove the second assertion of Theorem 4.1. Let us fix $s_{1}>0$, and let $\psi$ be a continuous function on $\mathbb{R}_{+}$with compact support contained in ]0, $\infty$ [. Let $F$ be a bounded continuous function on $\Omega$. It follows from Theorem 3.1 that

$$
\lim _{\varepsilon \rightarrow 0} \varepsilon^{-4} \mathbb{N}_{0}\left(\psi\left(\zeta_{s_{1}}\right) F(W) \mathbf{1}_{\{\underline{W}>-\varepsilon\}}\right)=\frac{2}{21} \mathbb{N}_{0}\left(\sigma^{-1} \psi\left(\zeta_{s_{1}}^{\left[s_{*}\right]}\right) F\left(W^{\left[s_{*}\right]}\right)\right)=\frac{2}{21} \overline{\mathbb{N}}_{0}\left(\psi\left(\zeta_{s_{1}}\right) F(W)\right) .
$$

To see this, apply Theorem 3.1 with a function $\varphi$ such that $s \varphi(s)$ vanishes on a neighborhood of 0 and is identically equal to 1 on $\left[s_{1}, \infty[\right.$.

On the other hand, we have also

$$
\begin{aligned}
\varepsilon^{-4} \mathbb{N}_{0}\left(\psi\left(\zeta_{s_{1}}\right) F(W) \mathbf{1}_{\{\underline{W}>-\varepsilon\}}\right) & =\varepsilon^{-4} \int_{0}^{\infty} \frac{\mathrm{d} h}{2 h^{2}} \mathbb{N}_{0}^{h}\left(\psi\left(\zeta_{s_{1}}\right) F(W) \mathbf{1}_{\{\underline{W}>-\varepsilon\}}\right) \\
& =\int_{0}^{\infty} \frac{\mathrm{d} h}{2 h^{2}} \varepsilon^{-4} \mathbb{N}_{0}^{h}(\underline{W}>-\varepsilon) \times \mathbb{N}_{0}^{h,(\varepsilon)}\left(\psi\left(\zeta_{s_{1}}\right) F(W)\right) .
\end{aligned}
$$

We pass to the limit $\varepsilon \rightarrow 0$ in the right-hand side of (35), using (26) and the first assertion of the theorem, which gives

$$
\lim _{\varepsilon \rightarrow 0} \mathbb{N}_{0}^{h,(\varepsilon)}\left(\psi\left(\zeta_{s_{1}}\right) F(W)\right)=\overline{\mathbb{N}}_{0}^{h}\left(\psi\left(\zeta_{s_{1}}\right) F(W)\right) .
$$


To justify dominated convergence, first note that

$$
\varepsilon^{-4} \mathbb{N}_{0}^{h}(\underline{W}>-\varepsilon)=\varepsilon^{-4} \mathbb{N}_{0}^{1}\left(\underline{W}>-\frac{\varepsilon}{\sqrt{h}}\right) \leqslant \frac{C}{h^{2}} .
$$

Furthermore, by comparing the intensity measures in (27) and (33), we get that the distribution of $\sigma$ under $\mathbb{N}_{0}^{h,(\varepsilon)}$ is stochastically bounded by the distribution of $\sigma$ under $\mathbb{N}_{0}^{h}$. Hence,

$$
\mathbb{N}_{0}^{h,(\varepsilon)}\left(\psi\left(\zeta_{s_{1}}\right) F(W)\right) \leqslant C^{\prime} \mathbb{N}_{0}^{h,(\varepsilon)}\left(\sigma>s_{1}\right) \leqslant C^{\prime} \mathbb{N}_{0}^{h}\left(\sigma>s_{1}\right) \leqslant C_{\left(s_{1}\right)} \exp \left(-\frac{C_{\left(s_{1}\right)}^{\prime}}{h^{2}}\right),
$$

where $C_{\left(s_{1}\right)}$ and $C_{\left(s_{1}\right)}^{\prime}$ are positive constants depending on $s_{1}$.

The previous observations allow us to apply the dominated convergence theorem to the right-hand side of (35), and to get

$$
\lim _{\varepsilon \downarrow 0} \varepsilon^{-4} \mathbb{N}_{0}\left(\psi\left(\zeta_{s_{1}}\right) F(W) \mathbf{1}_{\{\underline{W}>-\varepsilon\}}\right)=\frac{c_{0}}{2} \int_{0}^{\infty} \frac{\mathrm{d} h}{h^{4}} \overline{\mathbb{N}}_{0}^{h}\left(\psi\left(\zeta_{s_{1}}\right) F(W)\right) .
$$

Comparing with (34) now completes the proof.

At this point we have obtained two distinct descriptions of $\overline{\mathbb{N}}_{0}$ :

- The law of $\sigma$ under $\overline{\mathbb{N}}_{0}$ has density $(8 \pi)^{-1 / 2} s^{-5 / 2}$, and the conditional distribution $\overline{\mathbb{N}}_{0}(\cdot \mid \sigma=s)$ is the law under $\mathbb{N}_{0}^{(s)}$ of the re-rooted snake $W^{\left[s_{*}\right]}$.

- The law of $H$ under $\overline{\mathbb{N}}_{0}$ has density $\frac{21 c_{0}}{4} h^{-4}$, and the conditional distribution $\overline{\mathbb{N}}_{0}(\cdot \mid H=h)$ can be reconstructed from the "spine" $Y^{h}$ and the Poisson point measures $\mathcal{M}$ and $\mathcal{M}^{\prime}$ as explained in the proof of Theorem 4.1.

If we think of analogous results for the Itô measure of Brownian excursions, it is tempting to look for a more Markovian description of $\overline{\mathbb{N}}_{0}$. It is relatively easy to see that the process $\left(\left(\zeta_{s}, W_{s}\right), s>0\right)$ is Markovian under $\overline{\mathbb{N}}_{0}$, and to describe its transition kernels (informally, this is the Brownian snake conditioned not to exit $] 0, \infty[-$ compare with [2]). One would then like to have an explicit formula for entrance laws, that is for the law of $\left(\zeta_{s}, W_{s}\right)$ under $\overline{\mathbb{N}}_{0}$, for each fixed $s>0$. Such explicit expressions seem difficult to obtain. See however the calculations in Section 5.

In the final part of this section, we investigate the limiting behavior of the measures $\overline{\mathbb{N}}_{0}(\cdot \mid H=h)$ as $h \rightarrow \infty$. This leads to a (one-dimensional) Brownian snake conditioned to stay positive and to live forever. The motivation for introducing such a process comes from the fact that it is expected to appear in scaling limits of discrete trees coding random quadrangulations: See the recent work of Chassaing and Durhuus [9].

Before stating our result, we give a description of the limiting process. Let $Z=\left(Z_{t}, t \geqslant 0\right)$ be a nine-dimensional Bessel process started at 0 . Conditionally given $Z$, let

$$
\mathcal{P}=\sum_{i \in I} \delta_{\left(h_{i}, \omega_{i}\right)}
$$

be a Poisson point measure on $\mathbb{R}_{+} \times \Omega$ with intensity

$$
2 \mathbf{1}_{\{\mathcal{R}(\omega) \subset]-Z_{r}, \infty[\}} \mathrm{d} r \mathbb{N}_{0}(\mathrm{~d} \omega) .
$$

We may and will assume that $\mathcal{P}$ is constructed in the following way. Start from a Poisson point measure

$$
\mathcal{Q}=\sum_{j \in J} \delta_{\left(\bar{h}_{j}, \bar{\omega}_{j}\right)}
$$

with intensity $2 \mathrm{~d} r \mathbb{N}_{0}(\mathrm{~d} \omega)$, and assume that $\mathcal{Q}$ is independent of $Z$. Then set

$$
\mathcal{P}=\sum_{j \in J} \mathbf{1}_{\left\{\mathcal{R}\left(\bar{\omega}_{j}\right) \subset\right]-Z_{\bar{h}_{j}}, \infty[\}} \delta_{\left(\bar{h}_{j}, \bar{\omega}_{j}\right)} .
$$


We then construct our conditioned snake $W^{\infty}$ from the pair $(Z, \mathcal{P})$. This is very similar to the reconstruction mapping that was already used in the proof of Theorem 4.1. To simplify notation, we put

$$
\sigma_{i}=\sigma\left(\omega_{i}\right), \quad \zeta_{s}^{i}=\zeta_{s}\left(\omega_{i}\right), \quad W_{s}^{i}=W_{s}\left(\omega_{i}\right)
$$

for every $i \in I$ and $s \geqslant 0$. For every $\ell \in[0, h]$, we set

$$
\tau_{\ell}=\sum_{i \in I} \mathbf{1}_{\left\{h_{i} \leqslant \ell\right\}} \sigma_{i}
$$

Then, if $s \geqslant 0$, there is a unique $\ell$ such that $\tau_{\ell-} \leqslant s \leqslant \tau_{\ell}$, and:

- Either there is a (unique) $i \in I$ such that $\ell=h_{i}$, and we set

$$
\begin{aligned}
& \zeta_{s}^{\infty}=\ell+\zeta_{s-\tau_{\ell-},}^{i} \\
& W_{s}^{\infty}(t)= \begin{cases}Z_{t} & \text { if } t \leqslant \ell, \\
Z_{\ell}+W_{s-\tau_{\ell-}}^{i}(t-\ell) & \text { if } \ell<t \leqslant \zeta_{s}^{\infty} ;\end{cases}
\end{aligned}
$$

- Or there is no such $i$, and we set

$$
\begin{aligned}
& \zeta_{s}^{\infty}=\ell, \\
& W_{s}^{\infty}(t)=Z_{t}, \quad t \leqslant \ell .
\end{aligned}
$$

It is easy to verify that these prescriptions define a continuous process $W^{\infty}$ with values in $\mathcal{W}$. We denote by $\overline{\mathbb{N}}_{0}^{\infty}$ the law of $W^{\infty}$.

Theorem 4.3. The probability measures $\overline{\mathbb{N}}_{0}^{h}$ converge to $\overline{\mathbb{N}}_{0}^{\infty}$ when $h \rightarrow \infty$.

Proof. We rely on the explicit description of $\overline{\mathbb{N}}_{0}^{h}$ obtained in the proof of Theorem 4.1. Let $Y^{h}=\left(Y_{t}^{h}, 0 \leqslant t \leqslant h\right)$ be as in (32).

Lemma 4.4. The processes $\left(Y_{t \wedge h}^{h}, t \geqslant 0\right)$ converge in distribution to $Z$ as $h \rightarrow \infty$.

Proof. Let $A>0$ and let $\Phi$ be a bounded continuous function on $C\left([0, A], \mathbb{R}_{+}\right)$. By (32), if $h \geqslant A$,

$$
E\left[\Phi\left(Y_{t}^{h}, 0 \leqslant t \leqslant A\right)\right]=\frac{h^{2}}{c_{0}} E_{0}^{(9)}\left[\Phi\left(R_{t}, 0 \leqslant t \leqslant A\right) R_{h}^{-4} \exp \left(\int_{0}^{h} \frac{\mathrm{d} t}{R_{t}^{2}} G\left(\frac{R_{t}}{\sqrt{h-t}}\right)\right)\right] .
$$

We apply the Markov property at time $A$ in the right-hand side, and write $h=A+a$ to simplify notation:

$$
\begin{aligned}
E\left[\Phi\left(Y_{t}^{h}, 0 \leqslant t \leqslant A\right)\right]= & \frac{h^{2}}{c_{0}} E_{0}^{(9)}\left[\Phi\left(R_{t}, 0 \leqslant t \leqslant A\right) \exp \left(\int_{0}^{A} \frac{\mathrm{d} t}{R_{t}^{2}} G\left(\frac{R_{t}}{\sqrt{h-t}}\right)\right)\right. \\
& \times E_{R_{A}}^{(9)}\left[R_{a}^{-4} \exp \left(\int_{0}^{a} \frac{\mathrm{d} t}{R_{t}^{2}} G\left(\frac{R_{t}}{\sqrt{a-t}}\right)\right)\right] .
\end{aligned}
$$

From the bound $0 \leqslant G(x) \leqslant 2 x^{2}$, it is immediate that

$$
1 \leqslant \exp \left(\int_{0}^{A} \frac{\mathrm{d} t}{R_{t}^{2}} G\left(\frac{R_{t}}{\sqrt{h-t}}\right)\right) \leqslant\left(1+\frac{A}{a}\right)^{2} .
$$

On the other hand, a scaling argument gives

$$
\frac{h^{2}}{c_{0}} E_{R_{A}}^{(9)}\left[R_{a}^{-4} \exp \left(\int_{0}^{a} \frac{\mathrm{d} t}{R_{t}^{2}} G\left(\frac{R_{t}}{\sqrt{a-t}}\right)\right)\right]=\left(\frac{h}{a}\right)^{2} c_{0}^{-1} E_{R_{A} / \sqrt{a}}^{(9)}\left[R_{1}^{-4} \exp \left(\int_{0}^{1} \frac{\mathrm{d} t}{R_{t}^{2}} G\left(\frac{R_{t}}{\sqrt{1-t}}\right)\right)\right] .
$$


From (30), we know that

$$
\lim _{x \downarrow 0} E_{x}^{(9)}\left[R_{1}^{-4} \exp \left(\int_{0}^{1} \frac{\mathrm{d} t}{R_{t}^{2}} G\left(\frac{R_{t}}{\sqrt{1-t}}\right)\right)\right]=E_{0}^{(9)}\left[R_{1}^{-4} \exp \left(\int_{0}^{1} \frac{\mathrm{d} t}{R_{t}^{2}} G\left(\frac{R_{t}}{\sqrt{1-t}}\right)\right)\right]=c_{0} .
$$

We can use (38) and (39) to pass to the limit $h \rightarrow \infty$ in the right-hand side of (37). The justification of dominated convergence is easy thanks to the bounds we obtained when proving (30). It follows that

$$
\lim _{h \rightarrow \infty} E\left[\Phi\left(Y_{t}^{h}, 0 \leqslant t \leqslant A\right)\right]=E_{0}^{(9)}\left[\Phi\left(R_{t}, 0 \leqslant t \leqslant A\right)\right]
$$

which was the desired result.

We can now complete the proof of Theorem 4.3. By Lemma 4.4 and the Skorokhod representation theorem, we may assume that $\left(Y_{t \wedge h}^{h}\right)_{t \geqslant 0}$ converges to $\left(Z_{t}\right)_{t \geqslant 0}$ uniformly on every compact subset of $\mathbb{R}_{+}$, a.s.

Recall the description of $\overline{\mathbb{N}}_{0}^{h}$ as the law of $\Gamma\left(Y^{h}, \mathcal{M}, \mathcal{M}^{\prime}\right)$ in the proof of Theorem 4.1: According to this description, we can construct a process $\left(W_{s}^{h}\right)_{s \leqslant \alpha_{h}}$ having the distribution of $\left(W_{s}\right)_{s \leqslant \alpha}$ under $\overline{\mathbb{N}}_{0}^{h}$, by the same formulas we used to define $W^{\infty}$ from the pair $(Z, \mathcal{P})$, provided that $Z$ is replaced by $Y^{h}$, the point measure $\mathcal{P}$ is replaced by

$$
\mathcal{N}^{h}:=\sum_{j \in J} \mathbf{1}_{\left\{\mathcal{R}\left(\bar{\omega}_{j}\right) \subset\right]-Y_{\bar{h}_{j}}^{h}, \infty[\}} \mathbf{1}_{\left\{\bar{h}_{j}<h, H\left(\bar{\omega}_{j}\right)<h-\bar{h}_{j}\right\}} \delta_{\left(\bar{h}_{j}, \bar{\omega}_{j}\right)}
$$

(note that the conditional distribution of $\mathcal{N}^{h}$ knowing $Y^{h}$ is that of a Poisson point measure with intensity $\mu_{0}^{h}\left(Y^{h} ; \mathrm{d} r \mathrm{~d} \omega\right)$, as required) and we restrict our attention to

$$
s \leqslant \alpha_{h}:=\int \mathcal{N}^{h}(\mathrm{~d} r \mathrm{~d} \omega) \sigma(\omega) .
$$

When $h \rightarrow \infty$, the constraints $\left\{\bar{h}_{j}<h, H\left(\bar{\omega}_{j}\right)<h-\bar{h}_{j}\right\}$ in the definition of $\mathcal{N}^{h}$ play no role, and the convergence of $Y^{h}$ to $Z$ implies that $\mathcal{N}^{h}$ converges to $\mathcal{P}$, in a sense that can easily be made precise. It is then a straightforward exercise to verify that

$$
\lim _{h \rightarrow \infty}\left(W_{s \wedge \alpha_{h}}^{h}\right)_{s \geqslant 0}=\left(W_{s}^{\infty}\right)_{s \geqslant 0}
$$

uniformly on every compact subset of $\mathbb{R}_{+}$, a.s. The statement of Theorem 4.3 follows.

\section{Finite-dimensional marginal distributions under $\overline{\mathbb{N}}_{0}$}

Our goal in this section is to get an analogue of formula (1) when $\mathbb{N}_{x}$ is replaced by the conditional measure $\overline{\mathbb{N}}_{0}$. This result will be formally analogous to (1) but the role of Brownian motion for the spatial displacements will be played by the nine-dimensional Bessel process. More precisely, recall the notation before (1), and let $x \geqslant 0$. For a fixed marked tree $\theta=\left(\mathcal{T},\left(h_{u}\right)_{u \in \mathcal{T}}\right),\left(\xi^{u}, u \in \mathcal{T}\right)$ are independent linear Brownian motions under the probability measure $Q_{x}^{\theta}$. Under the same probability measure, we construct inductively a collection of nine-dimensional Bessel processes $\left(\xi^{u}, u \in \mathcal{T}\right)$ by first requiring that $\bar{\xi}^{\varnothing}$ is obtained as the solution of the stochastic differential equation

$$
\left\{\begin{array}{l}
\mathrm{d} \bar{\xi}_{t}^{\varnothing}=\mathrm{d} \xi_{t}^{\varnothing}+\frac{4}{\bar{\xi}_{t}^{\varnothing}} \mathrm{d} t, \quad 0 \leqslant t \leqslant h_{\varnothing} \\
\bar{\xi}_{0}^{\varnothing}=x
\end{array}\right.
$$

and then, for every $u \in \mathcal{T} \backslash\{\varnothing\}$, constructing $\bar{\xi}^{u}$ as the solution of

$$
\left\{\begin{array}{l}
\mathrm{d} \bar{\xi}_{t}^{u}=\mathrm{d} \xi_{t}^{u}+\frac{4}{\bar{\xi}_{t}^{u}} \mathrm{~d} t, \quad 0 \leqslant t \leqslant h_{u} \\
\bar{\xi}_{0}^{u}=\bar{\xi}_{h_{\pi(u)}(u)}
\end{array}\right.
$$

We then define $\left(\bar{V}_{a}, a \in \tilde{\theta}\right)$ by the formula $\bar{V}_{p_{\theta}(u, \ell)}=\bar{\xi}_{\ell}^{u}$ for every $u \in \mathcal{T}$ and $\ell \in\left[0, h_{u}\right]$. Finally, for every leaf $a$ of $\tilde{\theta}$, we define the stopped path $\overline{\mathrm{w}}^{(a)}$ from $\left(\bar{V}_{a}, a \in \tilde{\theta}\right)$ in the same way as $\mathrm{w}^{(a)}$ was defined from $\left(V_{a}, a \in \tilde{\theta}\right)$. Recall the notation $L(\theta)$ for the set of leaves of $\tilde{\theta}$, and $I(\theta)$ for the set of its nodes. 
Theorem 5.1. Let $p \geqslant 1$ be an integer. Let $F$ be a symmetric nonnegative measurable function on $\mathcal{W}^{p}$. Then,

$$
\overline{\mathbb{N}}_{0}\left(\int_{] 0, \sigma[p} \mathrm{d} s_{1} \cdots \mathrm{d} s_{p} F\left(W_{s_{1}}, \ldots, W_{s_{p}}\right)\right)=2^{p-1} p ! \int \Lambda_{p}(\mathrm{~d} \theta) Q_{0}^{\theta}\left[F\left(\left(\overline{\mathrm{w}}^{(a)}\right)_{a \in L(\theta)}\right) \prod_{a \in I(\theta)}\left(\bar{V}_{a}\right)^{4} \prod_{a \in L(\theta)}\left(\bar{V}_{a}\right)^{-4}\right] .
$$

Proof. We may assume that $F$ is continuous and bounded above by 1 , and that there exist positive constants $\delta$ and $M$ such that $F\left(\mathrm{w}_{1}, \ldots, \mathrm{w}_{p}\right)=0$ as soon as $\zeta_{\left(\mathrm{w}_{i}\right)} \notin[\delta, M]$ for some $i$. The proof will be divided in several steps.

Step 1. To simplify notation, we write $\mathcal{R}(V)=\left\{V_{a}, a \in \tilde{\theta}\right\}$, for the range of $V$, or equivalently for the union of the ranges of $\mathrm{w}^{(a)}$ for $a \in L(\theta)$. We first apply Theorem 2.2 to compute

$$
\begin{aligned}
& \mathbb{N}_{0}\left(\int_{] 0, \sigma[p} \mathrm{d} s_{1} \cdots \mathrm{d} s_{p} F\left(W_{s_{1}}, \ldots, W_{s_{p}}\right) \mathbf{1}_{\{\mathcal{R} \subset]-\varepsilon, \infty[\}}\right) \\
& =p ! 2^{p-1} \int \Lambda_{p}(\mathrm{~d} \theta) Q_{0}^{\theta}\left[F\left(\left(\mathrm{w}^{(a)}\right)_{a \in L(\theta)}\right) \mathbf{1}_{\{\mathcal{R}(V) \subset]-\varepsilon, \infty[\}} \exp \left(-4 \int \mathcal{L}_{\theta}(\mathrm{d} a) \mathbb{N}_{0}(\mathcal{R} \subset]-\varepsilon-V_{a}, \infty[)\right)\right] \\
& =p ! 2^{p-1} \int \Lambda_{p}(\mathrm{~d} \theta) Q_{0}^{\theta}\left[F\left(\left(\mathrm{w}^{(a)}\right)_{a \in L(\theta)}\right) \mathbf{1}_{\{\mathcal{R}(V) \subset]-\varepsilon, \infty[\}} \exp \left(-6 \int \frac{\mathcal{L}_{\theta}(\mathrm{d} a)}{\left(V_{a}+\varepsilon\right)^{2}}\right)\right] \\
& =p ! 2^{p-1} \int \Lambda_{p}(\mathrm{~d} \theta) Q_{\varepsilon}^{\theta}\left[F\left(\left(-\varepsilon+\mathrm{w}^{(a)}\right)_{a \in L(\theta)}\right) \mathbf{1}_{\{\mathcal{R}(V) \subset] 0, \infty[\}} \exp \left(-6 \int \frac{\mathcal{L}_{\theta}(\mathrm{d} a)}{\left(V_{a}\right)^{2}}\right)\right] .
\end{aligned}
$$

We then use Proposition 2.6 inductively to see that

$$
\begin{gathered}
\varepsilon^{-4} Q_{\varepsilon}^{\theta}\left[F\left(\left(-\varepsilon+\mathrm{w}^{(a)}\right)_{a \in L(\theta)}\right) \mathbf{1}_{\{\mathcal{R}(V) \subset] 0, \infty[\}} \exp \left(-6 \int \frac{\mathcal{L}_{\theta}(\mathrm{d} a)}{\left(V_{a}\right)^{2}}\right)\right] \\
=Q_{\varepsilon}^{\theta}\left[F\left(\left(-\varepsilon+\overline{\mathrm{w}}^{(a)}\right)_{a \in L(\theta)}\right) \prod_{a \in I(\theta)}\left(\bar{V}_{a}\right)^{4} \prod_{a \in L(\theta)}\left(\bar{V}_{a}\right)^{-4}\right] .
\end{gathered}
$$

We have thus proved that

$$
\begin{aligned}
& \varepsilon^{-4} \mathbb{N}_{0}\left(\int_{] 0, \sigma\left[^{p}\right.} \mathrm{d} s_{1} \cdots \mathrm{d} s_{p} F\left(W_{s_{1}}, \ldots, W_{s_{p}}\right) \mathbf{1}_{\{\underline{W}>-\varepsilon\}}\right) \\
& \quad=p ! 2^{p-1} \int \Lambda_{p}(\mathrm{~d} \theta) Q_{\varepsilon}^{\theta}\left[F\left(\left(-\varepsilon+\overline{\mathrm{w}}^{(a)}\right)_{a \in L(\theta)}\right) \prod_{a \in I(\theta)}\left(\bar{V}_{a}\right)^{4} \prod_{a \in L(\theta)}\left(\bar{V}_{a}\right)^{-4}\right] .
\end{aligned}
$$

Step 2. We focus on the right-hand side of (40). Our goal is to prove that

$$
\begin{gathered}
\lim _{\varepsilon \rightarrow 0} \int \Lambda_{p}(\mathrm{~d} \theta) Q_{\varepsilon}^{\theta}\left[F\left(\left(-\varepsilon+\overline{\mathrm{w}}^{(a)}\right)_{a \in L(\theta)}\right) \prod_{a \in I(\theta)}\left(\bar{V}_{a}\right)^{4} \prod_{a \in L(\theta)}\left(\bar{V}_{a}\right)^{-4}\right] \\
=\int \Lambda_{p}(\mathrm{~d} \theta) Q_{0}^{\theta}\left[F\left(\left(\overline{\mathrm{w}}^{(a)}\right)_{a \in L(\theta)}\right) \prod_{a \in I(\theta)}\left(\bar{V}_{a}\right)^{4} \prod_{a \in L(\theta)}\left(\bar{V}_{a}\right)^{-4}\right] .
\end{gathered}
$$

We first state a lemma.

Lemma 5.2. We have

$$
Q_{\varepsilon}^{\theta}\left[\prod_{a \in I(\theta)}\left(\bar{V}_{a}\right)^{4} \prod_{a \in L(\theta)}\left(\bar{V}_{a}\right)^{-4}\right] \leqslant E_{\varepsilon}^{(9)}\left[R_{D(\theta)}^{-4}\right]
$$

where $D(\theta)=\max \left\{d_{\theta}(0, a): a \in L(\theta)\right\}$.

Proof. We argue by induction on $p$. If $p=1$, the result is immediate, with an equality. Let $p \geqslant 2$ and let us assume that the result holds at order $1,2, \ldots, p-1$. Let $\theta=\left(\mathcal{T},\left(h_{u}, u \in \mathcal{T}\right)\right)$ be a marked tree with $p$ leaves. Write $h=h_{\varnothing}$. 
By decomposing $\theta$ at its first branching point, we get two marked trees $\theta^{\prime} \in \mathbb{T}_{j}$, and $\theta^{\prime \prime} \in \mathbb{T}_{p-j}$, for some $j \in$ $\{1, \ldots, p-1\}$, in such a way that

$$
\begin{aligned}
Q_{\varepsilon}^{\theta}\left[\prod_{a \in I(\theta)}\left(\bar{V}_{a}\right)^{4} \prod_{a \in L(\theta)}\left(\bar{V}_{a}\right)^{-4}\right] & =E_{\varepsilon}^{(9)}\left[R_{h}^{4} Q_{R_{h}}^{\theta^{\prime}}\left[\prod_{a \in I\left(\theta^{\prime}\right)}\left(\bar{V}_{a}\right)^{4} \prod_{a \in L\left(\theta^{\prime}\right)}\left(\bar{V}_{a}\right)^{-4}\right] Q_{R_{h}}^{\theta^{\prime \prime}}\left[\prod_{a \in I\left(\theta^{\prime \prime}\right)}\left(\bar{V}_{a}\right)^{4} \prod_{a \in L\left(\theta^{\prime \prime}\right)}\left(\bar{V}_{a}\right)^{-4}\right]\right] \\
& \leqslant E_{\varepsilon}^{(9)}\left[R_{h}^{4} E_{R_{h}}^{(9)}\left[R_{D\left(\theta^{\prime}\right)}^{-4}\right] E_{R_{h}}^{(9)}\left[R_{D\left(\theta^{\prime \prime}\right)}^{-4}\right]\right] .
\end{aligned}
$$

We have used the induction hypothesis in the last inequality. We now observe that $D(\theta)=h+\max \left\{D\left(\theta^{\prime}\right), D\left(\theta^{\prime \prime}\right)\right\}$. Assume for definiteness that $D(\theta)=h+D\left(\theta^{\prime}\right)$. Using the bound (4) and the Markov property we get

$$
E_{\varepsilon}^{(9)}\left[R_{h}^{4} E_{R_{h}}^{(9)}\left[R_{D\left(\theta^{\prime}\right)}^{-4}\right] E_{R_{h}}^{(9)}\left[R_{D\left(\theta^{\prime \prime}\right)}^{-4}\right]\right] \leqslant E_{\varepsilon}^{(9)}\left[E_{R_{h}}^{9}\left[R_{D\left(\theta^{\prime}\right)}^{-4}\right]\right]=E_{\varepsilon}^{(9)}\left[R_{h+D\left(\theta^{\prime}\right)}^{-4}\right]=E_{\varepsilon}^{(9)}\left[R_{D(\theta)}^{-4}\right]
$$

This completes the proof of the lemma.

As a consequence of Lemma 5.2, we get the bound

$$
\begin{aligned}
Q_{\varepsilon}^{\theta}\left[F\left(\left(-\varepsilon+\overline{\mathrm{w}}^{(a)}\right)_{a \in L(\theta)}\right) \prod_{a \in I(\theta)}\left(\bar{V}_{a}\right)^{4} \prod_{a \in L(\theta)}\left(\bar{V}_{a}\right)^{-4}\right] & \leqslant E_{\varepsilon}^{(9)}\left[R_{D(\theta)}^{-4}\right] \prod_{a \in L(\theta)} \mathbf{1}_{\left\{\delta \leqslant d_{\theta}(0, a) \leqslant M\right\}} \\
& \leqslant E_{0}^{(9)}\left[R_{D(\theta)}^{-4}\right] \prod_{a \in L(\theta)} \mathbf{1}_{\left\{\delta \leqslant d_{\theta}(0, a) \leqslant M\right\}} \\
& =\frac{E_{0}^{(9)}\left[R_{1}^{-4}\right]}{D(\theta)^{2}} \prod_{a \in L(\theta)} \mathbf{1}_{\left\{\delta \leqslant d_{\theta}(0, a) \leqslant M\right\}} .
\end{aligned}
$$

The last quantity is clearly integrable with respect to the measure $\Lambda_{p}(\mathrm{~d} \theta)$. In addition, using the continuity of $F$, it is easy to verify that

$$
\lim _{\varepsilon \rightarrow 0} Q_{\varepsilon}^{\theta}\left[F\left(\left(-\varepsilon+\overline{\mathrm{w}}^{(a)}\right)_{a \in L(\theta)}\right) \prod_{a \in I(\theta)}\left(\bar{V}_{a}\right)^{4} \prod_{a \in L(\theta)}\left(\bar{V}_{a}\right)^{-4}\right]=Q_{0}^{\theta}\left[F\left(\left(\overline{\mathrm{w}}^{(a)}\right)_{a \in L(\theta)}\right) \prod_{a \in I(\theta)}\left(\bar{V}_{a}\right)^{4} \prod_{a \in L(\theta)}\left(\bar{V}_{a}\right)^{-4}\right] .
$$

An application of the dominated convergence theorem now leads to (41).

Step 3. We now consider the left-hand side of formula (40). For every $0<b<1$, we consider the continuous function $\phi_{b}: \mathbb{R}_{+} \rightarrow[0,1]$ such that $\phi_{b}(s)=1$ for every $s \in[b, 1 / b], \phi_{b}(s)=0$ for every $\left.s \in \mathbb{R}_{+} \backslash\right] b / 2,2 / b\left[\right.$, and $\phi_{b}$ is linear on $[b / 2, b]$ and on $[1 / b, 2 / b]$. From Theorem 3.1 and the definition of $\overline{\mathbb{N}}_{0}$, we get

$$
\begin{aligned}
& \lim _{\varepsilon \rightarrow 0} \varepsilon^{-4} \mathbb{N}_{0}\left(\phi_{b}(\sigma) \int_{] 0, \sigma\left[^{p}\right.} \mathrm{d} s_{1} \cdots \mathrm{d} s_{p} F\left(W_{s_{1}}, \ldots, W_{s_{p}}\right) \mathbf{1}_{\{\underline{W}>-\varepsilon\}}\right) \\
& =\overline{\mathbb{N}}_{0}\left(\phi_{b}(\sigma) \int_{] 0, \sigma\left[^{p}\right.} \mathrm{d} s_{1} \cdots \mathrm{d} s_{p} F\left(W_{s_{1}}, \ldots, W_{s_{p}}\right)\right) .
\end{aligned}
$$

\section{Lemma 5.3. The following convergence holds:}

$$
\lim _{b \rightarrow 0} \sup _{\varepsilon \in(0,1)} \varepsilon^{-4} \mathbb{N}_{0}\left(\left(1-\phi_{b}(\sigma)\right) \int_{] 0, \sigma[p} \mathrm{d} s_{1} \cdots \mathrm{d} s_{p} F\left(W_{s_{1}}, \ldots, W_{s_{p}}\right) \mathbf{1}_{\{\underline{W}>-\varepsilon\}}\right)=0 .
$$

Proof. We first observe that

$$
\begin{aligned}
& \varepsilon^{-4} \mathbb{N}_{0}\left(\mathbf{1}_{\{\sigma<b\}} \int_{] 0, \sigma\left[^{p}\right.} \mathrm{d} s_{1} \cdots \mathrm{d} s_{p} F\left(W_{s_{1}}, \ldots, W_{s_{p}}\right) \mathbf{1}_{\{\underline{W}>-\varepsilon\}}\right) \\
& \leqslant b^{p} \varepsilon^{-4} \mathbb{N}_{0}\left(\sup _{s \in[0, \sigma]} \zeta_{s}>\delta, \underline{W}>-\varepsilon\right)=b^{p} \varepsilon^{-4} \int_{\delta}^{\infty} \frac{\mathrm{d} h}{2 h^{2}} \mathbb{N}_{0}^{h}(\underline{W}>-\varepsilon) \leqslant b^{p} \frac{C}{6 \delta^{3}}
\end{aligned}
$$


where the constant $C$ is such that $\varepsilon^{-4} \mathbb{N}_{0}^{h}(\underline{W}>-\varepsilon) \leqslant C h^{-2}$, for every $h>0$ and $0<\varepsilon<1$ (cf. (36)). On the other hand, the Cauchy-Schwarz inequality gives

$$
\begin{aligned}
& \varepsilon^{-4} \mathbb{N}_{0}\left(\mathbf{1}_{\{\sigma>1 / b\}} \int_{] 0, \sigma[p} \mathrm{d} s_{1} \cdots \mathrm{d} s_{p} F\left(W_{s_{1}}, \ldots, W_{s_{p}}\right) \mathbf{1}_{\{\underline{W}>-\varepsilon\}}\right) \\
& \quad \leqslant\left(\varepsilon^{-4} \mathbb{N}_{0}\left(\left(\int_{] 0, \sigma[p} \mathrm{d} s_{1} \cdots \mathrm{d} s_{p} F\left(W_{s_{1}}, \ldots, W_{s_{p}}\right)\right)^{2} \mathbf{1}_{\{\underline{W}>-\varepsilon\}}\right)\right)^{1 / 2}\left(\varepsilon^{-4} \mathbb{N}_{0}\left(\sigma>\frac{1}{b}, \underline{W}>-\varepsilon\right)\right)^{1 / 2} .
\end{aligned}
$$

Note that we may write

$$
\mathbb{N}_{0}\left(\left(\int_{] 0, \sigma\left[^{p}\right.} \mathrm{d} s_{1} \cdots \mathrm{d} s_{p} F\left(W_{s_{1}}, \ldots, W_{s_{p}}\right)\right)^{2} \mathbf{1}_{\{\underline{W}>-\varepsilon\}}\right)=\mathbb{N}_{0}\left(\int_{] 0, \sigma\left[^{2 p}\right.} \mathrm{d} s_{1} \cdots \mathrm{d} s_{2 p} G\left(W_{s_{1}}, \ldots, W_{s_{2 p}}\right) \mathbf{1}_{\{\underline{W}>-\varepsilon\}}\right),
$$

where $G$ is a nonnegative symmetric function on $\mathcal{W}^{2 p}$, which is also bounded by 1 . As a consequence of (40) and Lemma 5.2, we then get

$$
\begin{aligned}
& \varepsilon^{-4} \mathbb{N}_{0}\left(\left(\int_{] 0, \sigma[p} \mathrm{d} s_{1} \cdots \mathrm{d} s_{p} F\left(W_{s_{1}}, \ldots, W_{s_{p}}\right)\right)^{2} \mathbf{1}_{\{\underline{W}>-\varepsilon\}}\right) \\
& \quad \leqslant(2 p) ! 2^{2 p-1} E_{0}^{(9)}\left[R_{1}^{-4}\right] \int \Lambda_{2 p}(\mathrm{~d} \theta) D(\theta)^{-2} \prod_{a \in L(\theta)} \mathbf{1}_{\left\{\delta \leqslant d_{\theta}(\varnothing, a) \leqslant M\right\}}=C(p, \delta, M)<\infty .
\end{aligned}
$$

From Theorem 1.1 and a simple scaling argument, we have

$$
\varepsilon^{-4} \mathbb{N}_{0}\left(\sigma>\frac{1}{b}, \underline{W}>-\varepsilon\right)=\varepsilon^{-4} \int_{b^{-1}}^{\infty} \frac{\mathrm{d} s}{\sqrt{2 \pi s^{3}}} \mathbb{N}_{0}^{(s)}(\underline{W}>-\varepsilon) \leqslant C^{\prime} b^{1 / 2}
$$

By combining these estimates, we get

$$
\varepsilon^{-4} \mathbb{N}_{0}\left(\mathbf{1}_{\{\sigma>1 / b\}} \int_{] 0, \sigma\left[^{p}\right.} \mathrm{d} s_{1} \cdots \mathrm{d} s_{p} F\left(W_{s_{1}}, \ldots, W_{s_{p}}\right) \mathbf{1}_{\{\underline{W}>-\varepsilon\}}\right) \leqslant\left(C^{\prime} C(p, \delta, M)\right)^{1 / 2} b^{1 / 4} .
$$

Lemma 5.3 follows from (43) and (44).

We can now complete the proof of Theorem 5.1. First, by monotone convergence,

$$
\lim _{b \rightarrow 0} \overline{\mathbb{N}}_{0}\left(\phi_{b}(\sigma) \int_{] 0, \sigma\left[^{p}\right.} \mathrm{d} s_{1} \cdots \mathrm{d} s_{p} F\left(W_{s_{1}}, \ldots, W_{s_{p}}\right)\right)=\overline{\mathbb{N}}_{0}\left(\int_{] 0, \sigma[p} \mathrm{d} s_{1} \cdots \mathrm{d} s_{p} F\left(W_{s_{1}}, \ldots, W_{s_{p}}\right)\right) .
$$

From (42) and Lemma 5.3, it then follows that

$$
\lim _{\varepsilon \rightarrow 0} \varepsilon^{-4} \mathbb{N}_{0}\left(\int_{] 0, \sigma[p} \mathrm{d} s_{1} \cdots \mathrm{d} s_{p} F\left(W_{s_{1}}, \ldots, W_{s_{p}}\right) \mathbf{1}_{\{\underline{W}>-\varepsilon\}}\right)=\overline{\mathbb{N}}_{0}\left(\int_{] 0, \sigma[p} \mathrm{d} s_{1} \cdots \mathrm{d} s_{p} F\left(W_{s_{1}}, \ldots, W_{s_{p}}\right)\right) .
$$

Combining this with (40) and (41) gives Theorem 5.1.

\section{Acknowledgement}

The first author wishes to thank Philippe Chassaing for a stimulating conversation which motivated the present work. We also thank the referee for several useful remarks. 


\section{References}

[1] R. Abraham, W. Werner, Avoiding probabilities for Brownian snakes and super-Brownian motion, Electron. J. Probab. 2 (3) (1997) 27.

[2] R. Abraham, L. Serlet, Representations of the Brownian snake with drift, Stochastics Stochastics Rep. 73 (2002) $287-308$.

[3] D. Aldous, The continuum random tree I, Ann. Probab. 19 (1991) 1-28.

[4] D. Aldous, The continuum random tree. II. An overview, in: Stochastic Analysis, Durham, 1990, in: London Math. Soc. Lecture Note Ser., vol. 167, Cambridge Univ. Press, Cambridge, 1991, pp. 23-70.

[5] D. Aldous, The continuum random tree III, Ann. Probab. 21 (1993) 248-289.

[6] D. Aldous, Tree-based models for random distribution of mass, J. Statist. Phys. 73 (1993) 625-641.

[7] J. Bouttier, P. Di Francesco, E. Guitter, Random trees between two walls: exact partition function, J. Phys. A 36 (2003) $12349-12366$.

[8] J. Bouttier, P. Di Francesco, E. Guitter, Statistics of planar graphs viewed from a vertex: a study via labeled trees, Nucl. Phys. B 675 (2003) $631-660$

[9] P. Chassaing, B. Durhuus, Statistical Hausdorff dimension of labelled trees and quadrangulations, Preprint, 2003.

[10] P. Chassaing, G. Schaeffer, Random planar lattices and integrated super-Brownian excursion, Probab. Theory Related Fields 128 (2004) $161-212$.

[11] R. Cori, B. Vauquelin, Planar trees are well labeled trees, Canad. J. Math. 33 (1981) 1023-1042.

[12] J.F. Delmas, Computation of moments for the length of the one dimensional ISE support, Electron. J. Probab. 8 (17) (2003) 15.

[13] E. Derbez, G. Slade, The scaling limit of lattice trees in high dimensions, Comm. Math. Phys. 198 (1998) 69-104.

[14] T. Duquesne, A limit theorem for the contour process of conditioned Galton-Watson trees, Ann. Probab. 31 (2003) 996-1027.

[15] T. Duquesne, J.F. Le Gall, Probabilistic and fractal aspects of Lévy trees, Probab. Theory Related Fields 131 (2005) $553-603$.

[16] S.N. Evans, J.W. Pitman, A. Winter, Rayleigh processes, real trees and root growth with re-grafting, Probab. Theory Related Fields (2003), in press.

[17] T. Hara, G. Slade, The scaling limit of the incipient infinite cluster in high-dimensional percolation. II. Integrated super-Brownian excursion. Probabilistic techniques in equilibrium and nonequilibrium statistical physics, J. Math. Phys. 41 (2000) 1244-1293.

[18] S. Janson, J.F. Marckert, Convergence of discrete snakes, J. Theoret. Probab. (2003), in press.

[19] K.M. Jansons, L.C.G. Rogers, Decomposing the branching Brownian path, Ann. Probab. 2 (1992) 973-986.

[20] O. Kallenberg, Random Measures, Academic Press, London, 1975.

[21] J.F. Le Gall, Brownian excursions, trees and measure-valued branching processes, Ann. Probab. 19 (1991) 1399-1439.

[22] J.F. Le Gall, The uniform random tree in a Brownian excursion, Probab. Theory Related Fields 96 (1993) 369-383.

[23] J.F. Le Gall, The Brownian snake and solutions of $\Delta u=u^{2}$ in a domain, Probab. Theory Related Fields 102 (1995) $393-432$.

[24] J.F. Le Gall, Spatial Branching Processes, Random Snakes and Partial Differential Equations, Lectures Math. ETH Zürich, Birkhäuser, Boston, 1999.

[25] J.F. Le Gall, A conditional limit theorem for tree-indexed random walk, Stochastic Process. Appl. (2005), in press.

[26] J.F. Marckert, A. Mokkadem, State spaces of the snake and its tour - convergence of the discrete snake, J. Theoret. Probab. 16 (2004) 10151046.

[27] J.F. Marckert, A. Mokkadem, Limits of normalized quadrangulations. The Brownian map, Preprint, 2004.

[28] D. Revuz, M. Yor, Continuous Martingales and Brownian Motion, Springer, Berlin, 1991.

[29] R. van der Hofstad, G. Slade, Convergence of critical oriented percolation to super-Brownian motion above $4+1$ dimensions, Ann. Inst. H. Poincaré Probab. Statist. 39 (2003) 413-485.

[30] W. Vervaat, A relation between Brownian bridge and Brownian excursion, Ann. Probab. 7 (1979) 143-149.

[31] M. Yor, Loi de l'indice du lacet brownien, et distribution de Hartman-Watson, Z. Wahr. Verw. Gebiete 53 (1980) 71-95. 